\title{
A Full Visible Spectrum Panchromatic Triple Donor Dye for Dye-Sensitized Solar Cells
}

Jonathon Watson, Taylor Santaloci, Hammad Cheema, Ryan C. Fortenberry, and Jared

H. Delcamp*

Coulter Hall, Department of Chemistry and Biochemistry, University of Mississippi,

University, MS, 38677, United States.

*corresponding author email: delcamp@olemiss.edu 


\section{TABLE OF CONTENTS:}

1. Optical and Electrochemical data (Figures S1-S3, Table S1) S3-S5

2. JW1 Device Optimization Studies (Figures S4-S10, Tables S2-S3) S6-S10

3. Charge extraction studies (Figure S11)

$\mathrm{S} 11$

4. Computational studies (Tables S4-S5, Figure S12-S17) S12-S17

5. Computational studies (XYZ coordinates) S18-S31

6. NMR spectra for intermediates and final dye (Figure S18-S27) S32-S41 


\section{Optical and Electrochemical Data}

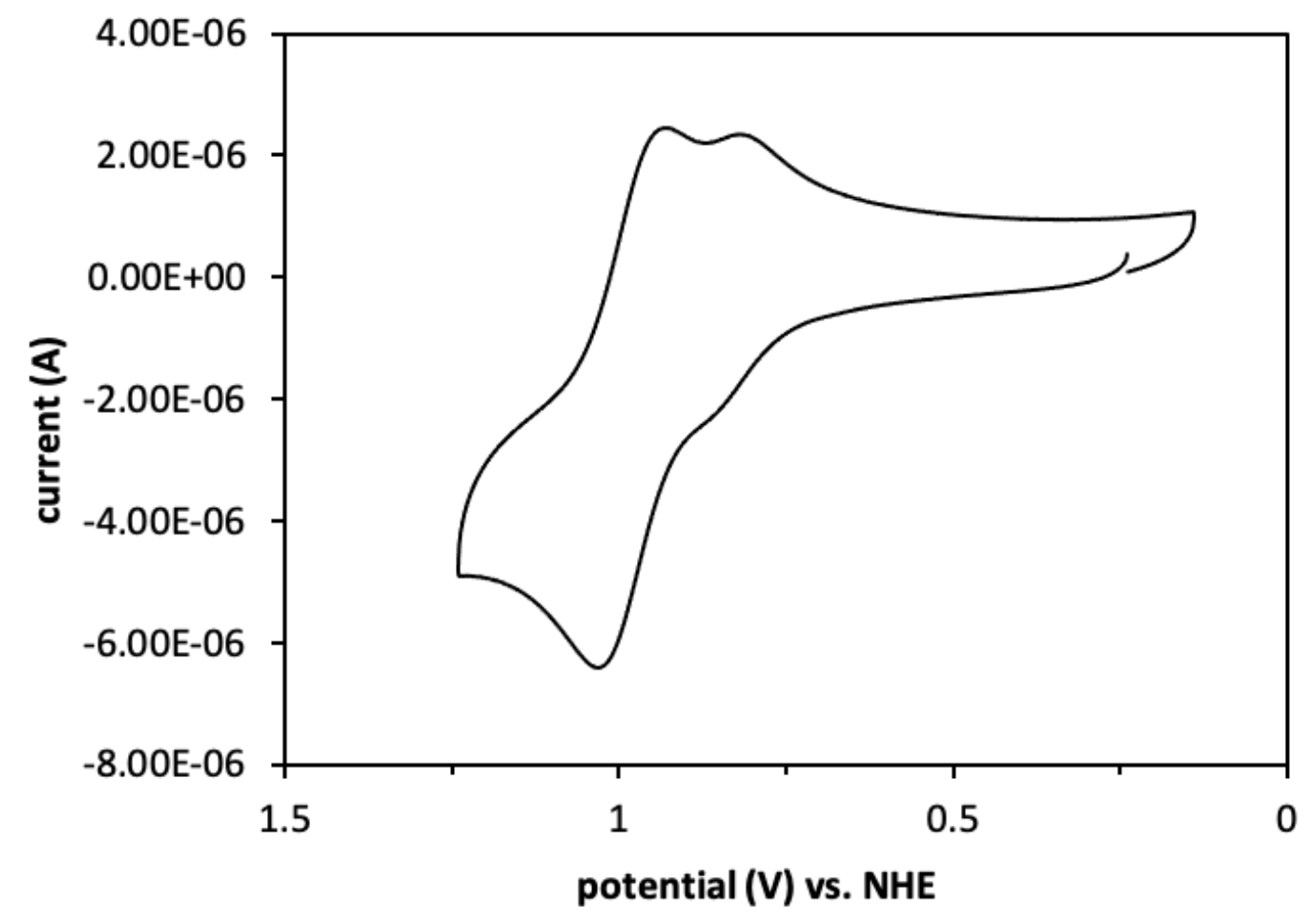

Figure S1. Cyclic voltammogram collected for JW1 in dichloromethane solution with 0.1 $\mathrm{M} \mathrm{Bu}_{4} \mathrm{NPF}_{6}$ as electrolyte, platinum counter electrode, $\mathrm{Ag} / \mathrm{AgCl}$ reference electrode and a glassy carbon working electrode. Ferrocene was used as a reference standard, taken as 0.70 $\mathrm{V}$ versus NHE in DCM and oxidation potentials are reported versus normal hydrogen electrode (NHE). 


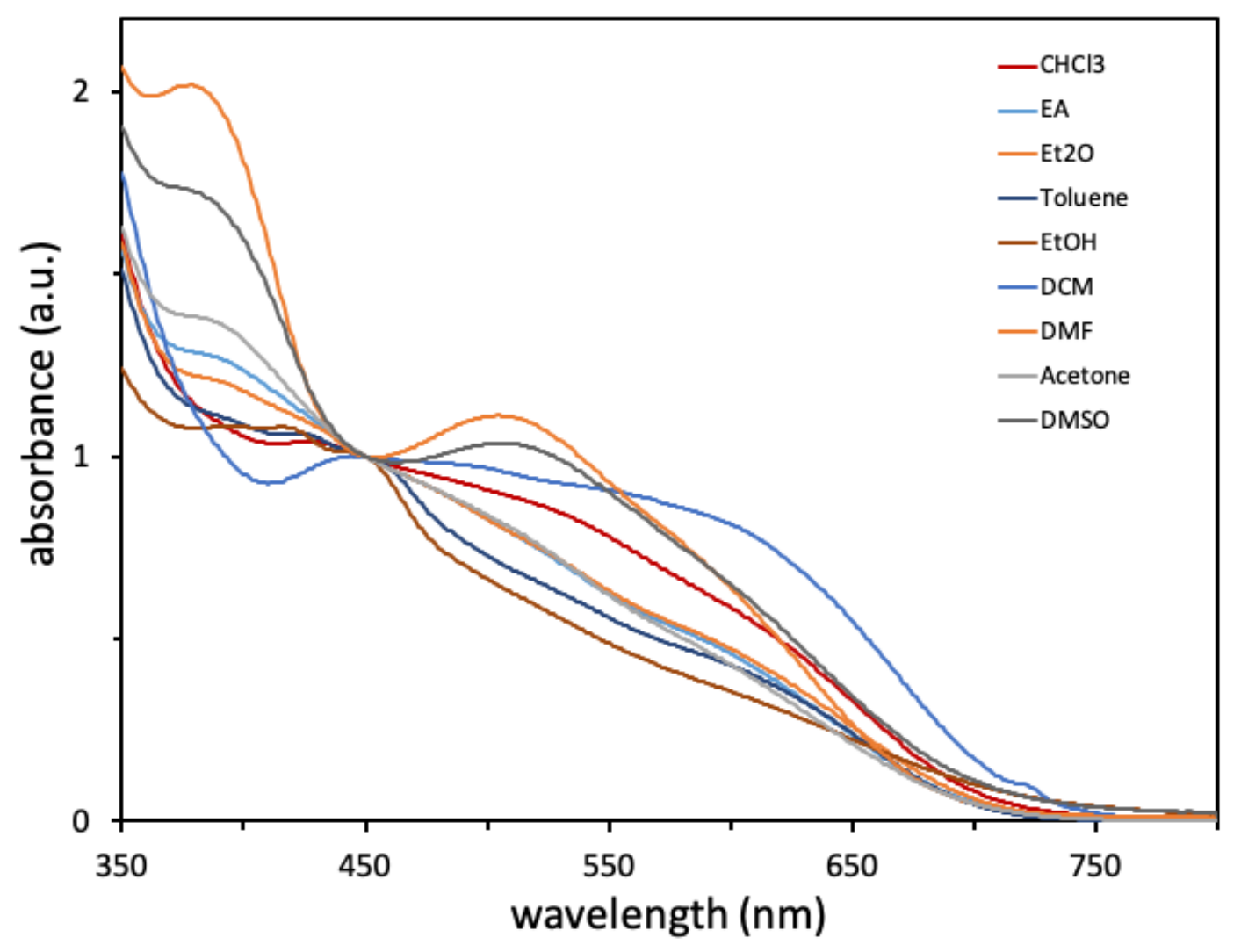

Figure S2. UV/Vis/NIR absorption spectra of $\mathbf{J W 1}$ in various solvents. $\mathrm{CHCl}_{3}$ is chloroform, EA is ethyl acetate, $\mathrm{Et}_{2} \mathrm{O}$ is diethyl ether, $\mathrm{EtOH}$ is ethanol, DCM is dichloromethane, DMF is $N, N$-dimethylformamide, and DMSO is dimethyl sulfoxide. 


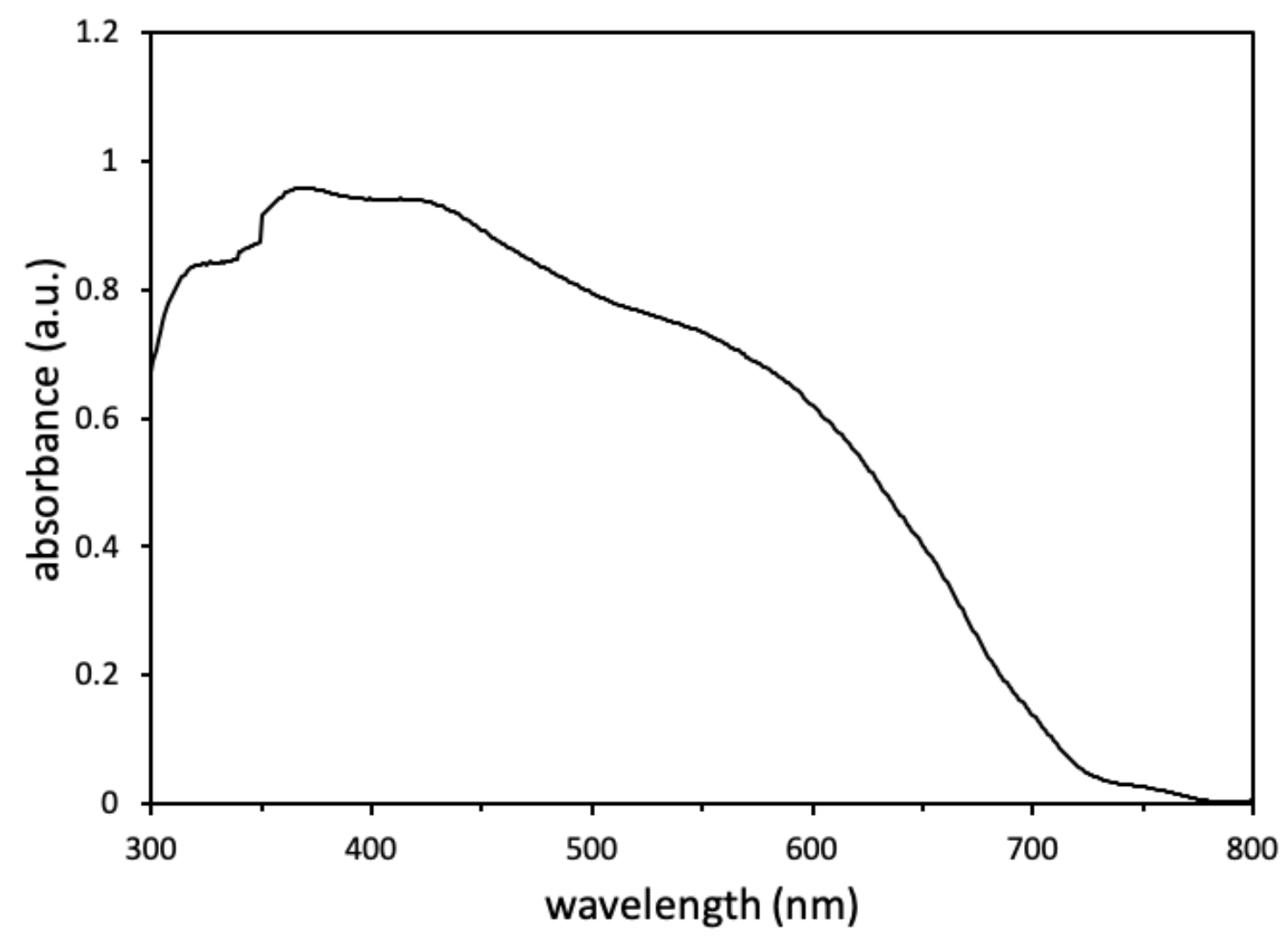

Figure S3. Absorption spectrum for $\mathbf{J W 1}$ on the $\mathrm{TiO}_{2}$ surface. The sensitization solution used was $0.3 \mathrm{mM}$ dye in 7:3 $\mathrm{EtOH}: \mathrm{CHCl}_{3}$ (10x CDCA). Electrode thickness was 3 micrometers of transparent $\mathrm{TiO}_{2}$.

Table S1. Dye loading studies for JW1 for various solvents versus NL6.

\begin{tabular}{|c|c|c|c|c|}
\hline $\begin{array}{l}\text { Dye } \\
\text { dipping solvent }\end{array}$ & $\begin{array}{l}\text { JW1 } \\
8: 2 \\
\text { EtOH:THF }\end{array}$ & $\begin{array}{l}\text { JW1 } \\
\text { 7:3 EtOH: } \\
\mathrm{CHCl}_{3}\end{array}$ & $\begin{array}{l}\text { JW1 } \\
\text { 7:3 EtOH: } \mathrm{CHCl}_{3} \\
\text { (10x CDCA) }\end{array}$ & $\begin{array}{l}\text { NL6 } \\
7: 3 \\
\text { EtOH:CHCl }{ }_{3} \\
\text { (10x CDCA) }\end{array}$ \\
\hline $\begin{array}{l}\text { dye loading } \\
\left(\mathrm{mol} / \mathrm{cm}^{2}\right)\end{array}$ & $1.14 \times 10^{-7}$ & $1.06 \times 10^{-7}$ & $1.03 \times 10^{-7}$ & $1.10 \times 10^{-7}$ \\
\hline
\end{tabular}




\section{JW1 device optimization studies}

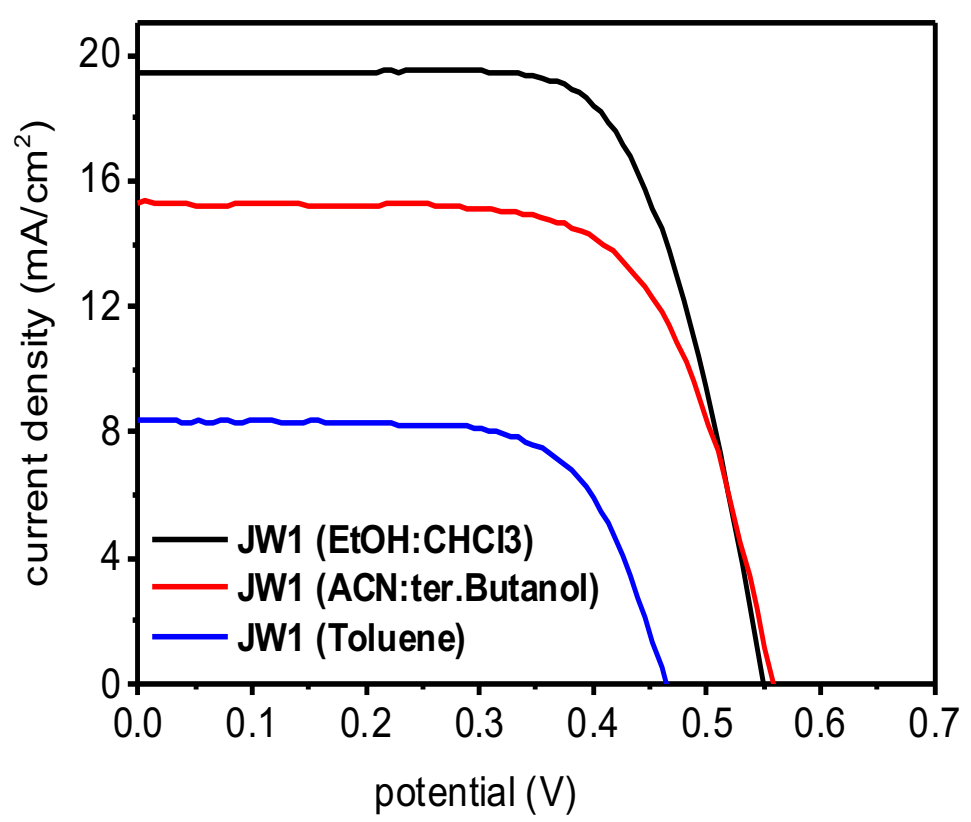

Figure S4. $J$ - $V$ curve presenting sensitization solvent effects on JW1 device performance. 10x CDCA is added to the EtOH: $\mathrm{CHCl}_{3}$ and $\mathrm{ACN}$ :tert-butanol solutions. Solubility limits preclude the use of CDCA in this amount with toluene.

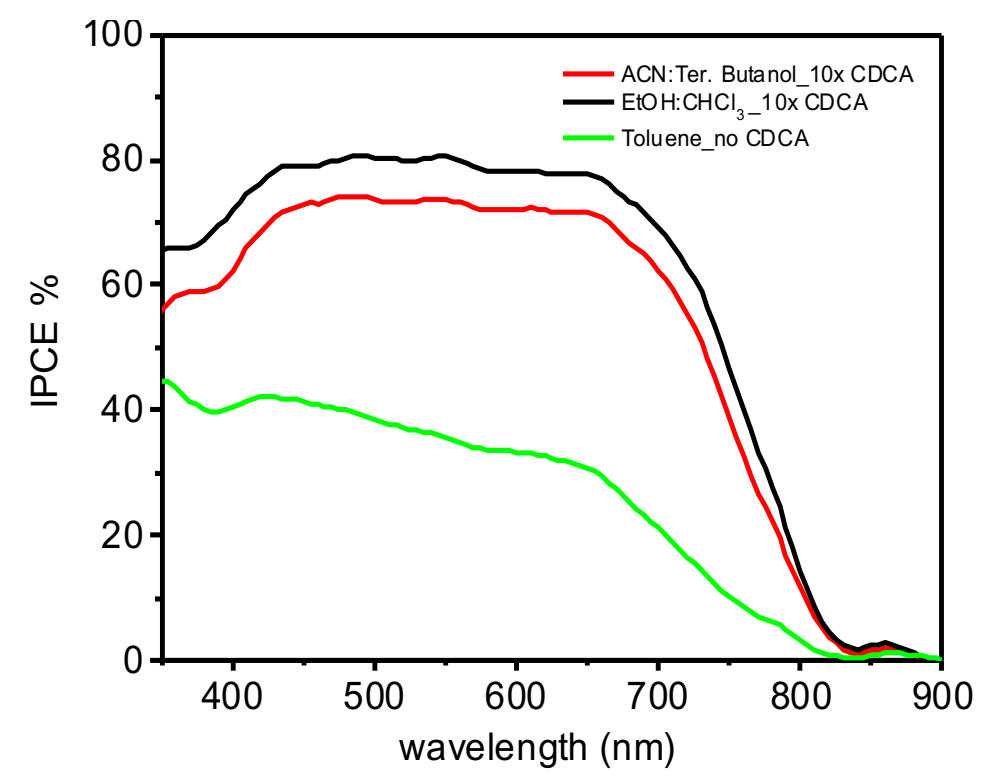

Figure S5. IPCE curve presenting sensitization solvent effects on JW1 performance. 


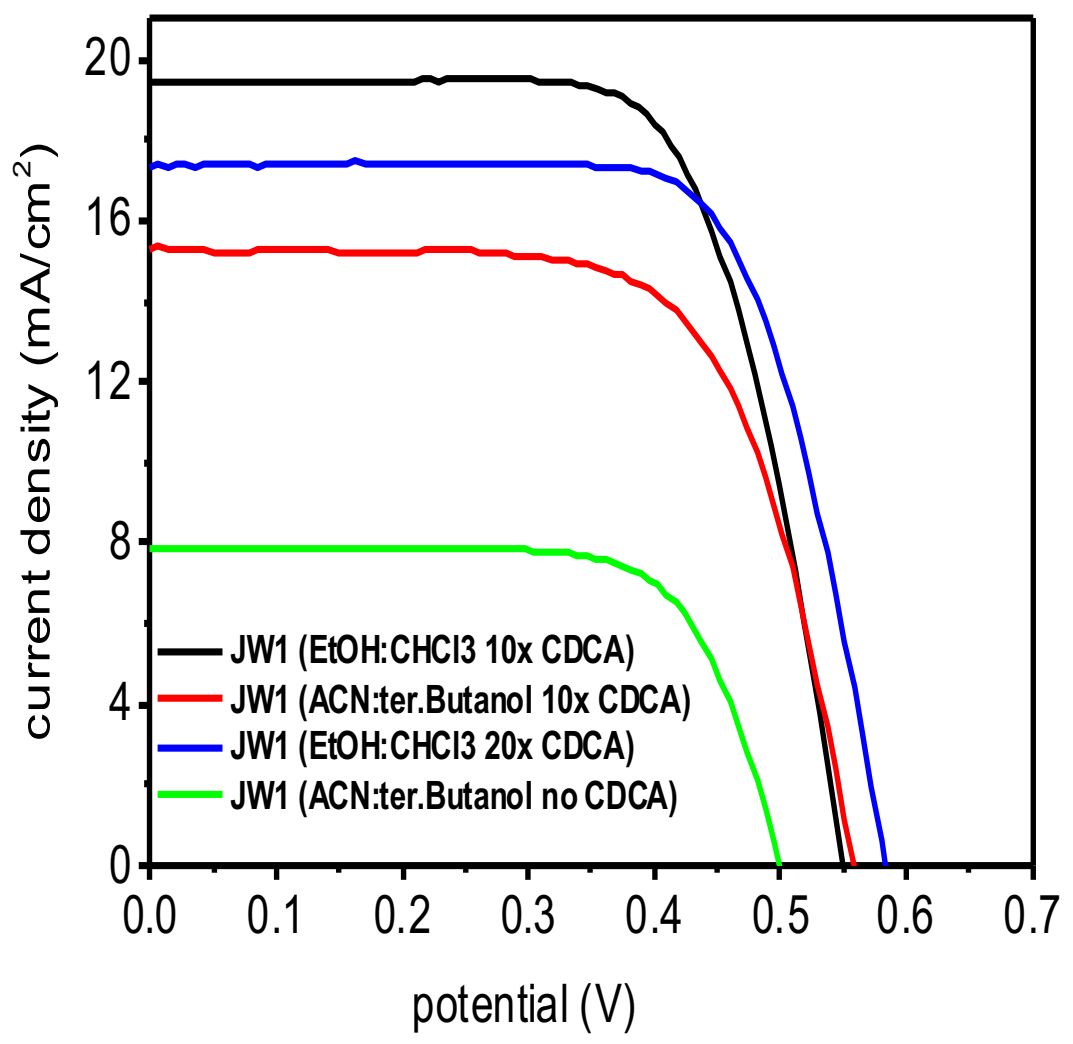

Figure S6. $J-V$ curve illustrating CDCA concentration effects on JW1 device performance. 


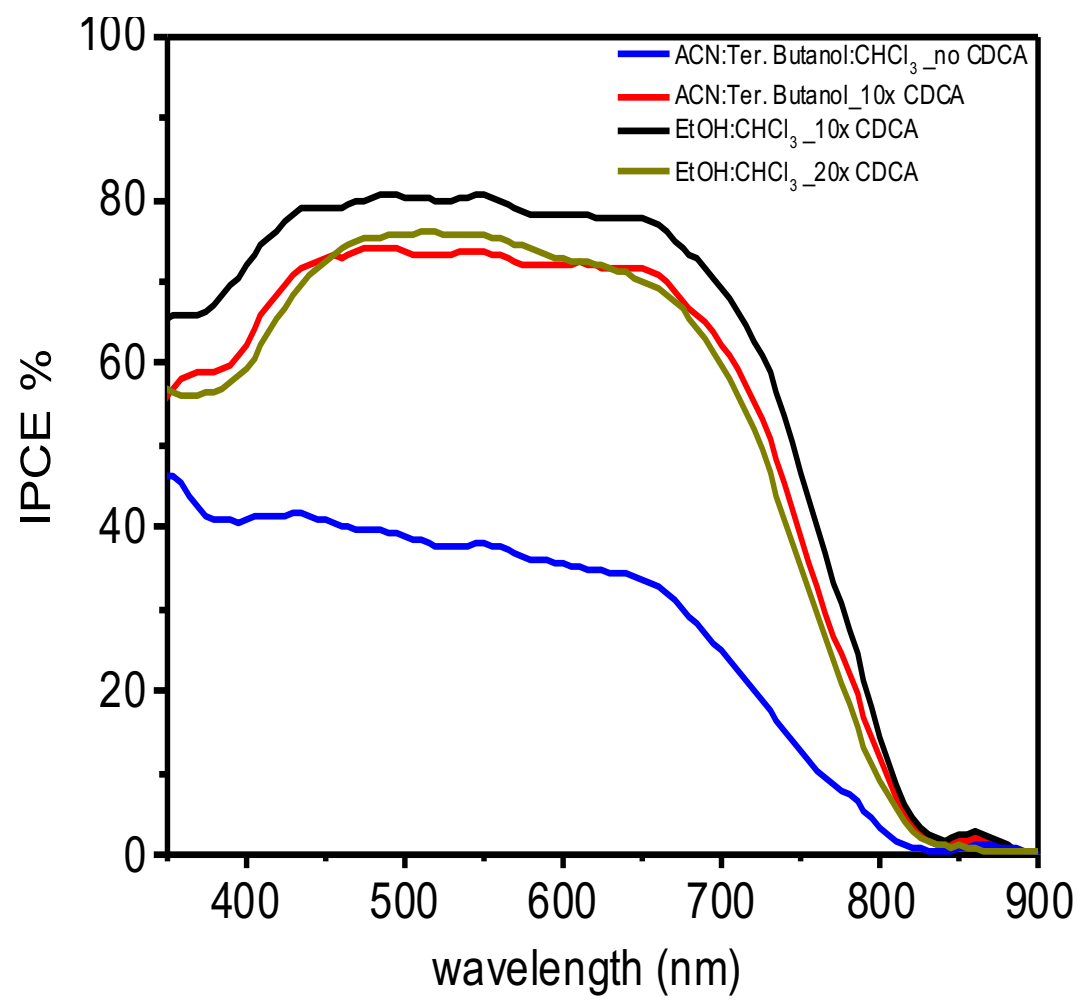

Figure S7. IPCE curve illustrating CDCA concentration effects on JW1 device performance.

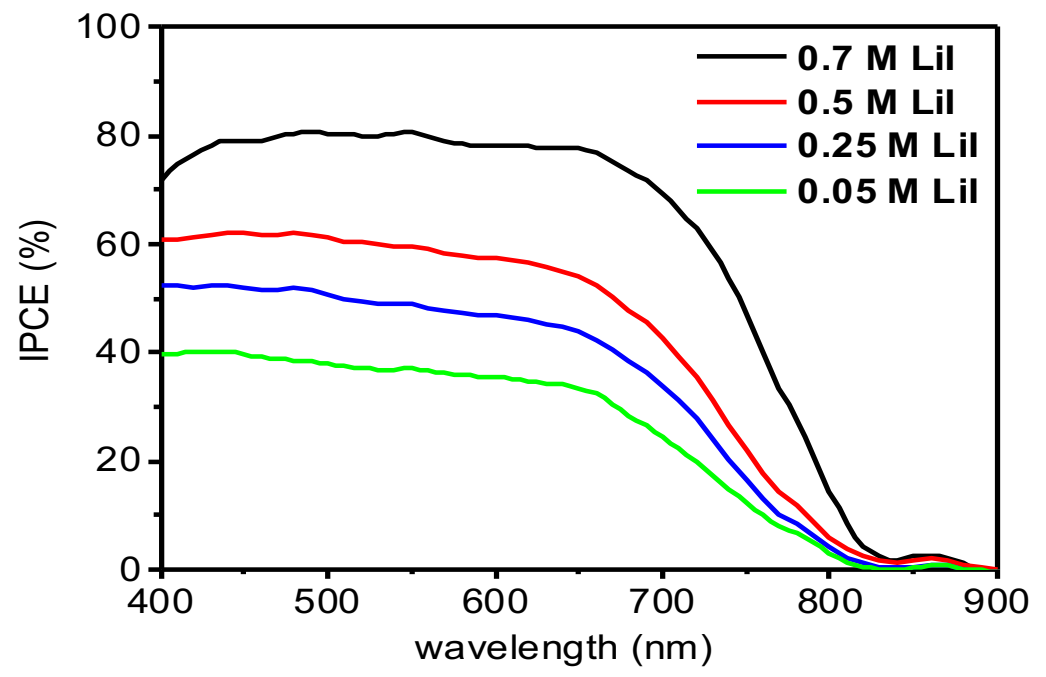

Figure S8. Effects of LiI concentration in the electrolyte on JW1 device performance. Solvent: EtOH: $\mathrm{CHCl}_{3}$ (7:3) with $0.3 \mathrm{mM}$ dye, 10x CDCA, dipping overnight at room temperature. 


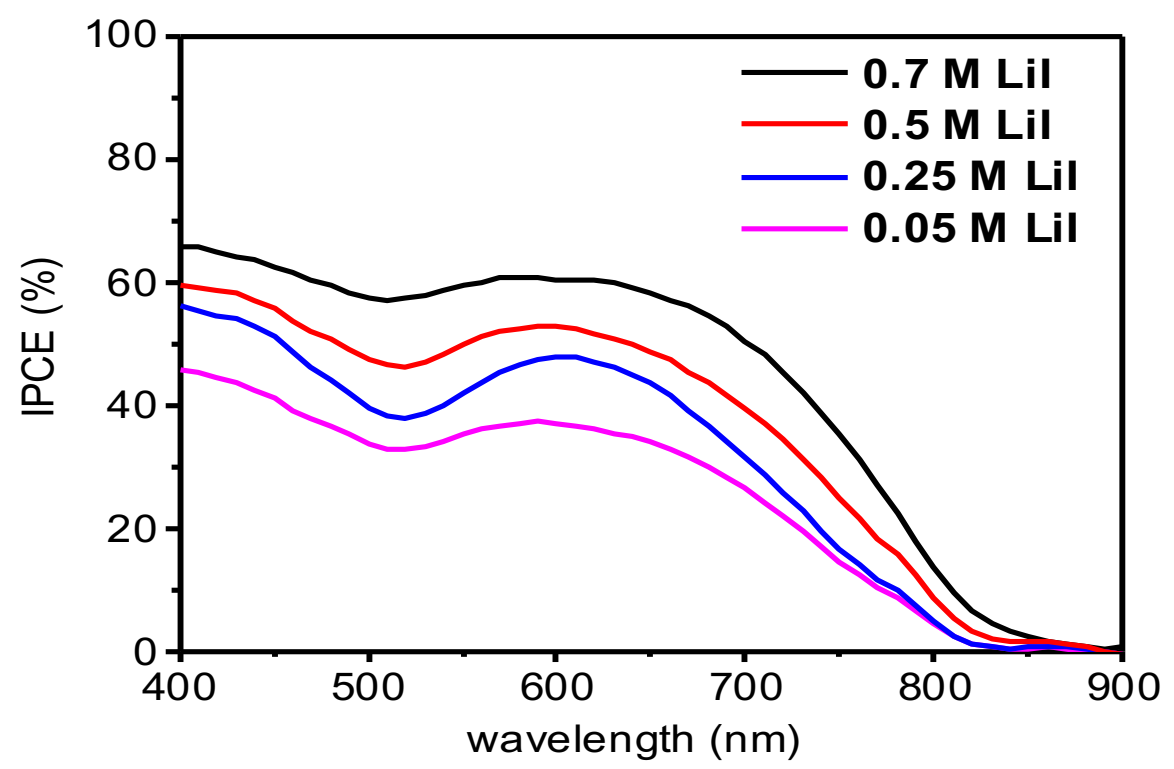

Figure S9. Effects of LiI concentration in the electrolyte on NL6 device performance. Solvent: $\mathrm{EtOH}: \mathrm{CHCl}_{3}$ (7:3) with $0.3 \mathrm{mM}$ dye, 10x CDCA, dipping overnight at room temperature.

Table S2. Results for LiI concentration studies.

\begin{tabular}{|l|l|l|l|l|l|}
\hline & LiI & $V_{\text {oc }}(\mathrm{mV})$ & $J_{\text {sc }}\left(\mathrm{mA} / \mathrm{cm}^{2}\right)$ & FF (\%) & PCE (\%) \\
\hline JW1 & $0.05 \mathrm{M}$ & 573 & 8.7 & 74 & 3.7 \\
\hline & $0.25 \mathrm{M}$ & 545 & 12.1 & 70 & 4.6 \\
\hline & $0.5 \mathrm{M}$ & 532 & 14.5 & 65 & 5.2 \\
\hline & $0.7 \mathrm{M}$ & 558 & 19.7 & 67 & 7.3 \\
\hline & $1.0 \mathrm{M}$ & 551 & 19.7 & 69 & 7.4 \\
\hline & & & & & \\
\hline NL6 & $0.05 \mathrm{M}$ & 538 & 9.2 & 69 & 3.5 \\
\hline & $0.25 \mathrm{M}$ & 525 & 11.3 & 68 & 4.1 \\
\hline & $0.5 \mathrm{M}$ & 486 & 12.8 & 64 & 4.0 \\
\hline & $0.7 \mathrm{M}$ & 561 & 15.0 & 64 & 5.3 \\
\hline & $1.0 \mathrm{M}$ & 606 & 13.6 & 66 & 5.4 \\
\hline
\end{tabular}




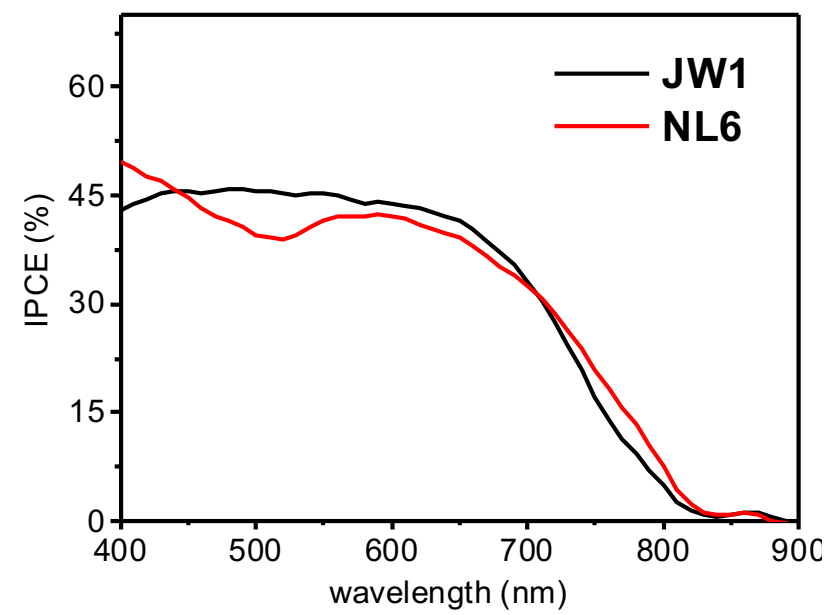

Figure S10. Effects of film thickness on device performance $(5 \mu \mathrm{m}$ active layer $+5 \mu \mathrm{m}$ scattering layer $\mathrm{TiO}_{2}$ ).

Table S3. Effects of film thickness on device performance ( $5 \mu \mathrm{m}$ active layer $+5 \mu \mathrm{m}$ scattering layer).

\begin{tabular}{|l|l|l|l|l|l|}
\hline dye & $\begin{array}{l}\text { active }+ \text { scattering } \\
\text { layer } \mathrm{TiO}_{2} \text { thickness }\end{array}$ & $\begin{array}{l}\text { Voc } \\
(\mathrm{mV})\end{array}$ & $\begin{array}{l}\text { Jsc } \\
\left(\mathrm{mA} / \mathrm{cm}^{2}\right)\end{array}$ & $\begin{array}{l}\mathrm{FF} \\
(\%)\end{array}$ & $\begin{array}{l}\text { PCE } \\
(\%)\end{array}$ \\
\hline JW1 & $5+5$ & 601 & 10.8 & 61.0 & 3.9 \\
\hline NL6 & $5+5$ & 580 & 10.7 & 58.0 & 3.7 \\
\hline
\end{tabular}




\section{Charge extraction studies}

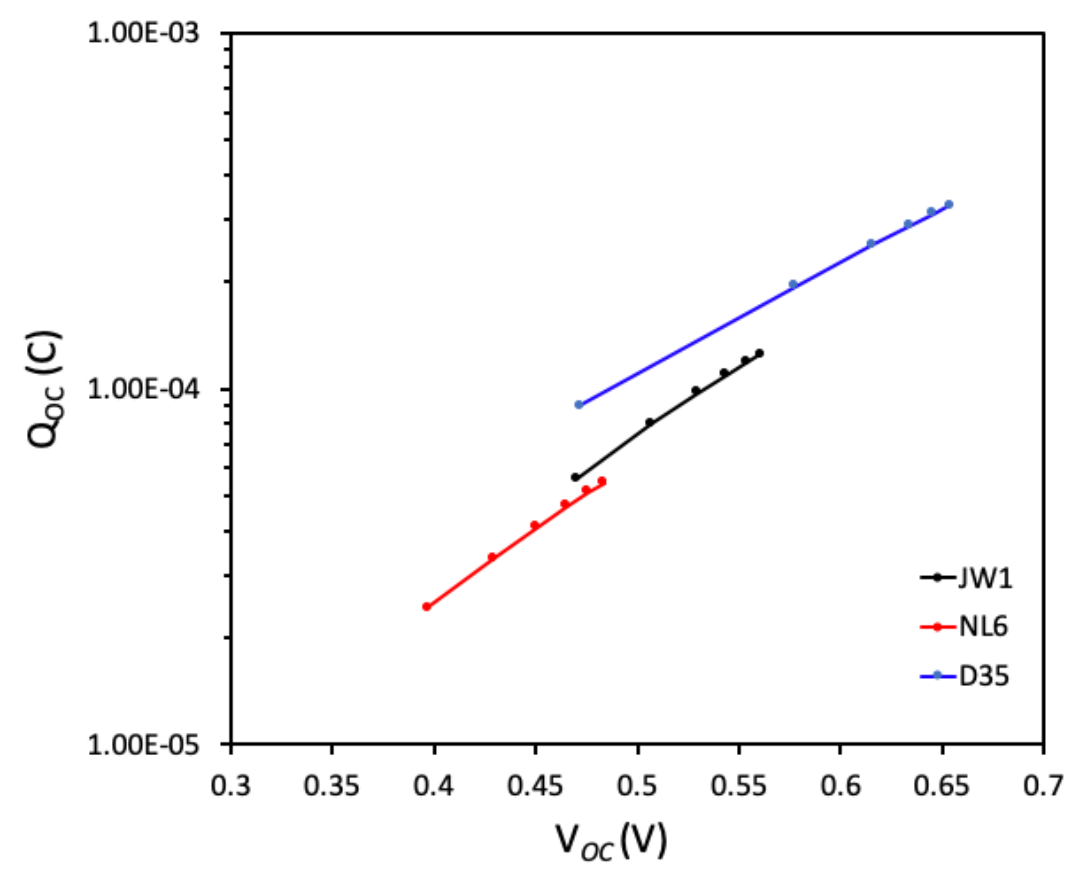

Figure S11. Charge extraction (Qoc) measurement for JW1, NL6, and D35 as a benchmark reference. (See J. Am. Chem. Soc. 2010, 132, 16714 for details about D35.) 


\section{Computational Studies}

General Computational Details: Time-dependent density functional theory (TD-DFT) computations were performed with optimized geometries and with the B3LYP functional and $6-311 \mathrm{G}(\mathrm{d}, \mathrm{p})$ basis set to compute the vertical transition energies and oscillator strengths both in isolation and dichloromethane solvent. All alkyl chains were truncated to simple methyl groups. Frequency calculations reveal no imaginary frequencies.

Table S4. Summary of transitions for JW1 and NL6 obtained from TD-DFT calculations in isolation using B3LYP/6-311G(d,p) above $400 \mathrm{~nm}$ with $f>0.1$.

\begin{tabular}{|c|c|c|c|c|c|c|c|}
\hline & State & Orbitals & Contribution & $\begin{array}{l}\text { Vert. Trans. } \\
(\mathrm{nm} \mid \mathrm{eV})\end{array}$ & $\begin{array}{l}\text { Oscillator } \\
\text { strength }\end{array}$ & $\begin{array}{l}\text { Exp. } \lambda_{\max } \\
(\mathrm{nm} \mid \mathrm{eV})\end{array}$ & $\begin{array}{l}\text { Exp. } \varepsilon\left(\mathrm{M}^{-}\right. \\
\left.{ }^{1} \mathrm{~cm}^{-1}\right)\end{array}$ \\
\hline \multirow[t]{3}{*}{ NL6 } & S1 & $\mathrm{H} \rightarrow \mathrm{L}$ & $97 \%$ & $708 \mid 1.75$ & 0.6971 & $605 \mid 2.05$ & 17,000 \\
\hline & S3 & $\begin{array}{l}\mathrm{H}-1 \rightarrow \mathrm{L} \\
\mathrm{H} \rightarrow \mathrm{L}+1\end{array}$ & $\begin{array}{l}79 \% \\
17 \%\end{array}$ & $485 \mid 2.56$ & 0.3790 & & \\
\hline & S4 & $\begin{array}{l}\mathrm{H}-2 \rightarrow \mathrm{L} \\
\mathrm{H}-5 \rightarrow \mathrm{L} \\
\mathrm{H} \rightarrow \mathrm{L}+2\end{array}$ & $\begin{array}{l}83 \% \\
5 \% \\
4 \%\end{array}$ & 409 | 3.03 & 0.1182 & & \\
\hline \multirow[t]{7}{*}{ JW1 } & S1 & $\begin{array}{l}H \rightarrow L \\
H-1 \rightarrow L\end{array}$ & $\begin{array}{l}94 \% \\
4 \%\end{array}$ & 689 | 1.80 & 0.5350 & $590 \mid 2.10$ & 20,100 \\
\hline & S2 & $\begin{array}{l}\mathrm{H}-1 \rightarrow \mathrm{L} \\
\mathrm{H} \rightarrow \mathrm{L}\end{array}$ & $\begin{array}{l}95 \% \\
4 \%\end{array}$ & $671 \mid 1.85$ & 0.1981 & & \\
\hline & S3 & $\mathrm{H}-2 \rightarrow \mathrm{L}$ & $98 \%$ & $617 \mid 2.01$ & 0.2338 & & \\
\hline & S4 & $\begin{array}{l}\mathrm{H} \rightarrow \mathrm{L}+1 \\
\mathrm{H}-3 \rightarrow \mathrm{L}\end{array}$ & $\begin{array}{l}86 \% \\
11 \%\end{array}$ & $518 \mid 2.39$ & 0.1355 & & \\
\hline & S5 & $\begin{array}{l}\mathrm{H}-1 \rightarrow \mathrm{L}+1 \\
\mathrm{H}-3 \rightarrow \mathrm{L}\end{array}$ & $\begin{array}{l}86 \% \\
12 \%\end{array}$ & $496 \mid 2.50$ & 0.1209 & & \\
\hline & S6 & $\begin{array}{l}\mathrm{H}-3 \rightarrow \mathrm{L} \\
\mathrm{H}-2 \rightarrow \mathrm{L}+1 \\
\mathrm{H}-1 \rightarrow \mathrm{L}+1 \\
\mathrm{H} \rightarrow \mathrm{L}+1\end{array}$ & $\begin{array}{l}63 \% \\
14 \% \\
10 \% \\
9 \%\end{array}$ & $476 \mid 2.61$ & 0.4198 & & \\
\hline & $\mathrm{S} 10$ & $\begin{array}{l}H-3 \rightarrow L \\
H \rightarrow L+2 \\
H-9 \rightarrow L \\
H-4 \rightarrow L\end{array}$ & $\begin{array}{l}82 \% \\
5 \% \\
4 \% \\
3 \%\end{array}$ & $389 \mid 3.19$ & 0.1436 & & \\
\hline
\end{tabular}

* State transitions are listed when the oscillator strength is $>0.1$ and the vertical transition is greater than $\sim 400 \mathrm{~nm}$. 
Table S5. Summary of transitions for JW1 and NL6 obtained from TD-DFT calculations using B3LYP/6-311G(d,p) above $400 \mathrm{~nm}$ with $f>0.1$ in dichloromethane as implicit solvent.

\begin{tabular}{|c|c|c|c|c|c|c|c|}
\hline & State & Orbitals & Contribution & $\begin{array}{l}\text { Vert. Trans. } \\
(\mathrm{nm} \mid \mathrm{eV})\end{array}$ & $\begin{array}{l}\text { Oscillator } \\
\text { strength }\end{array}$ & $\begin{array}{l}\text { Exp. } \lambda_{\max } \\
(\mathrm{nm} \mid \mathrm{eV})\end{array}$ & $\begin{array}{l}\text { Exp. } \varepsilon\left(M^{-}\right. \\
\left.{ }^{1} \mathrm{~cm}^{-1}\right)\end{array}$ \\
\hline \multirow[t]{4}{*}{ NL6 } & S1 & $\mathrm{H} \rightarrow \mathrm{L}$ & $99 \%$ & 755 | 1.64 & 0.9158 & $605 \mid 2.05$ & 17,000 \\
\hline & S3 & $\begin{array}{l}\mathrm{H}-1 \rightarrow \mathrm{L} \\
\mathrm{H} \rightarrow \mathrm{L}+1\end{array}$ & $\begin{array}{l}89 \% \\
9 \%\end{array}$ & $504 \mid 2.46$ & 0.4204 & & \\
\hline & S4 & $\begin{array}{l}\mathrm{H}-3 \rightarrow \mathrm{L} \\
\mathrm{H}-1 \rightarrow \mathrm{L}+1\end{array}$ & $\begin{array}{l}87 \% \\
6 \%\end{array}$ & 419 | 2.96 & 0.1712 & & \\
\hline & S5 & $\begin{array}{l}H-1 \rightarrow L+1 \\
H-5 \rightarrow L \\
H-3 \rightarrow L \\
H-5 \rightarrow L+1\end{array}$ & $\begin{array}{l}68 \% \\
14 \% \\
6 \% \\
3 \%\end{array}$ & 412 | 3.01 & 0.2102 & & \\
\hline \multirow[t]{7}{*}{ JW1 } & S1 & $\mathrm{H} \rightarrow \mathrm{L}$ & $97 \%$ & 749 | 1.66 & 0.6393 & $590 \mid 2.10$ & 20,100 \\
\hline & S2 & $\mathrm{H}-1 \rightarrow \mathrm{L}$ & $97 \%$ & $711 \mid 1.74$ & 0.2810 & & \\
\hline & S3 & $\mathrm{H}-2 \rightarrow \mathrm{L}$ & $98 \%$ & $663 \mid 1.87$ & 0.2714 & & \\
\hline & S4 & $\begin{array}{l}\mathrm{H} \rightarrow \mathrm{L}+1 \\
\mathrm{H}-3 \rightarrow \mathrm{L}\end{array}$ & $\begin{array}{l}94 \% \\
2 \%\end{array}$ & $547 \mid 2.26$ & 0.2443 & & \\
\hline & S5 & $\begin{array}{l}\mathrm{H}-1 \rightarrow \mathrm{L}+1 \\
\mathrm{H}-3 \rightarrow \mathrm{L}\end{array}$ & $\begin{array}{l}77 \% \\
21 \%\end{array}$ & $518 \mid 2.40$ & 0.1242 & & \\
\hline & S6 & $\begin{array}{l}\mathrm{H}-3 \rightarrow \mathrm{L} \\
\mathrm{H}-2 \rightarrow \mathrm{L}+1 \\
\mathrm{H}-1 \rightarrow \mathrm{L}+1 \\
\mathrm{H} \rightarrow \mathrm{L}+1\end{array}$ & $\begin{array}{l}51 \% \\
33 \% \\
11 \% \\
2 \%\end{array}$ & $496 \mid 2.50$ & 0.3840 & & \\
\hline & S10 & $\begin{array}{l}\mathrm{H}-3 \rightarrow \mathrm{L}+1 \\
\mathrm{H}-10 \rightarrow \mathrm{L}\end{array}$ & $\begin{array}{l}83 \% \\
8 \%\end{array}$ & $400 \mid 3.10$ & 0.1153 & & \\
\hline
\end{tabular}

*State transitions are listed when the oscillator strength is $>0.1$ and the vertical transition is greater than $\sim 400 \mathrm{~nm}$. 


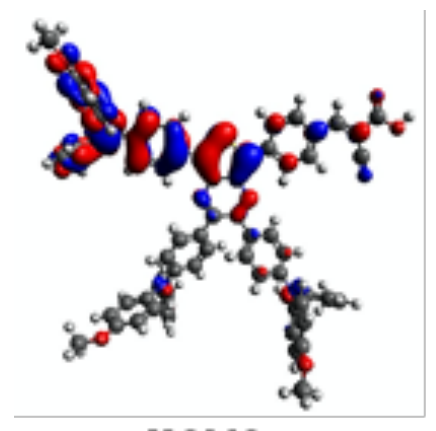

HOMO

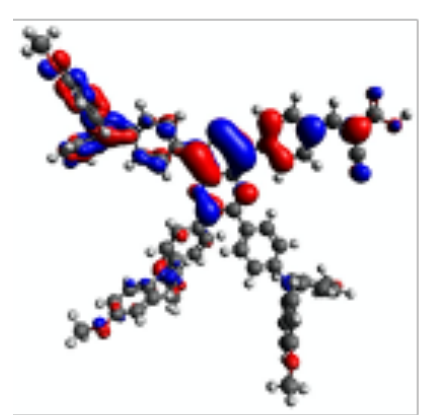

HOMO-3

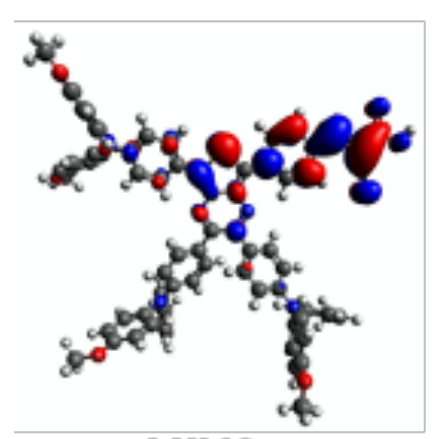

LUMO

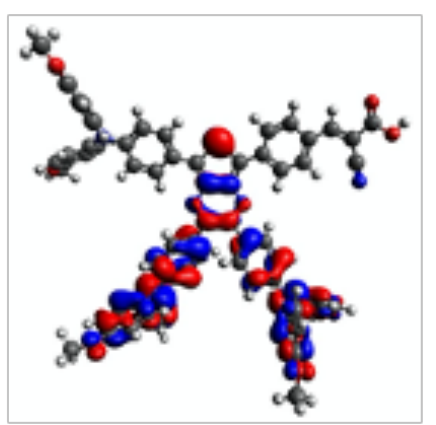

HOMO-1

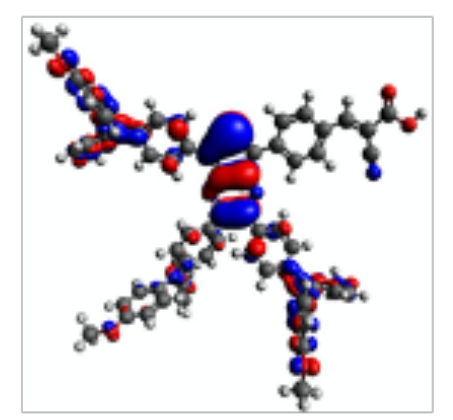

HOMO-4

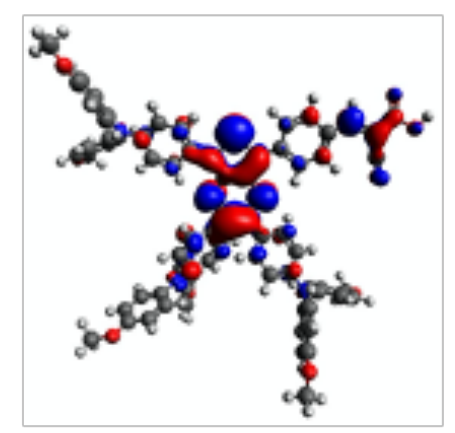

LUMO+1

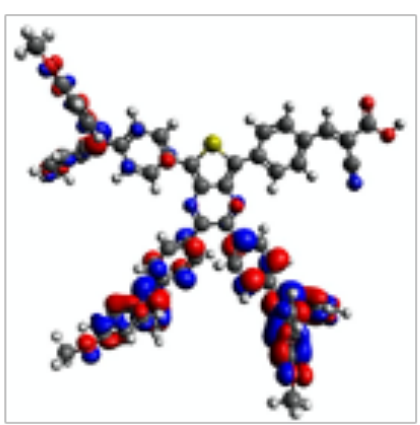

HOMO-2

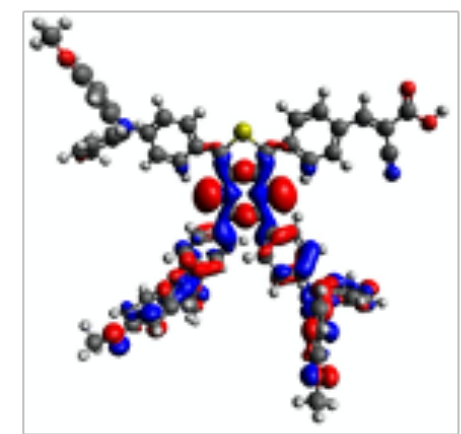

HOMO-9

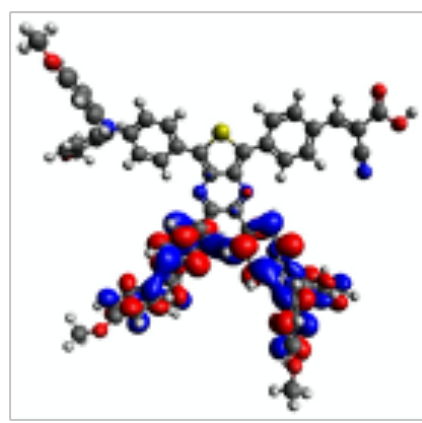

LUMO+2

Figure S12. Orbitals for JW1 (isolation). 

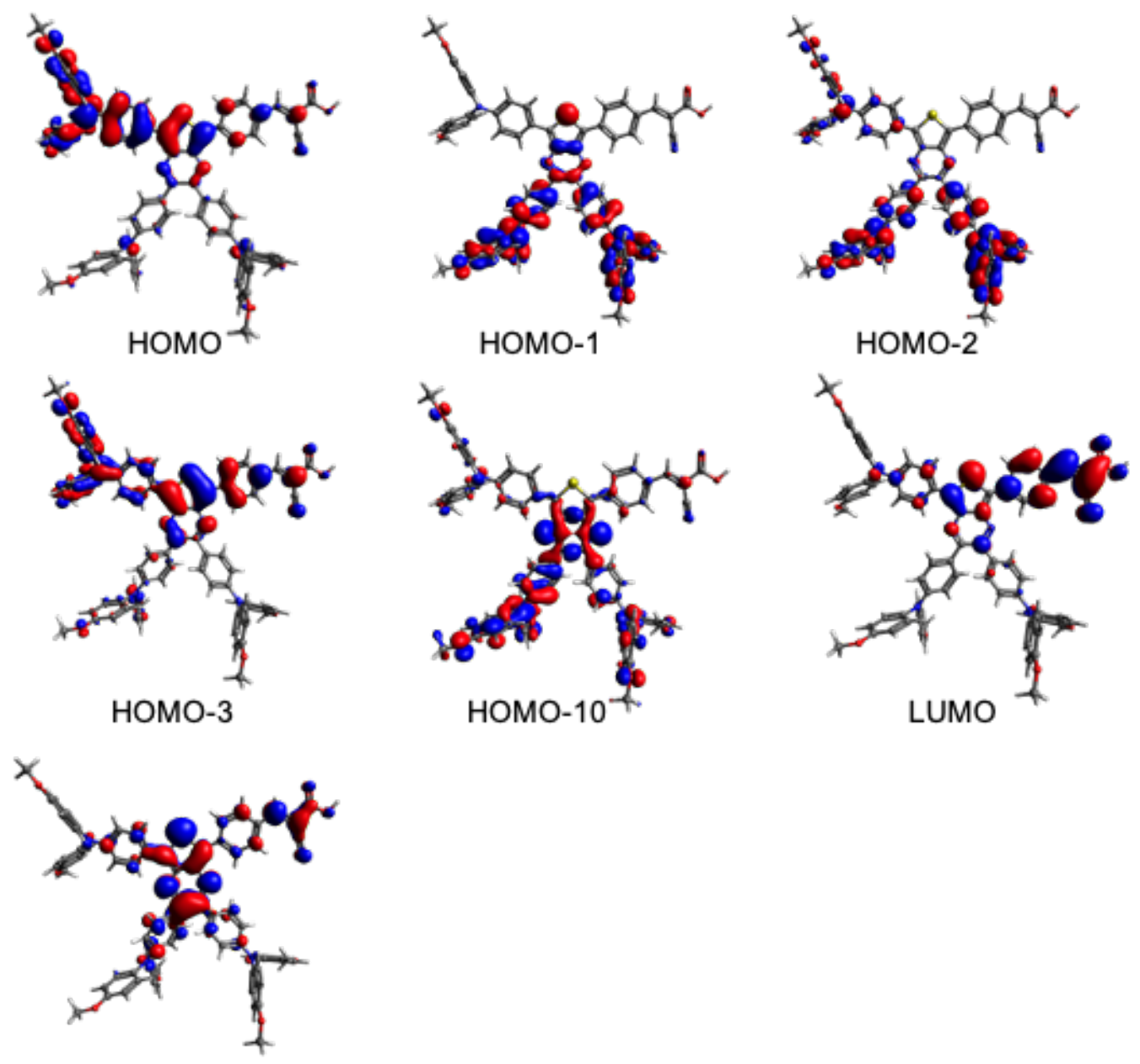

LUMO+1

Figure S13. Orbitals for JW1 (with DCM as implicit solvent). 


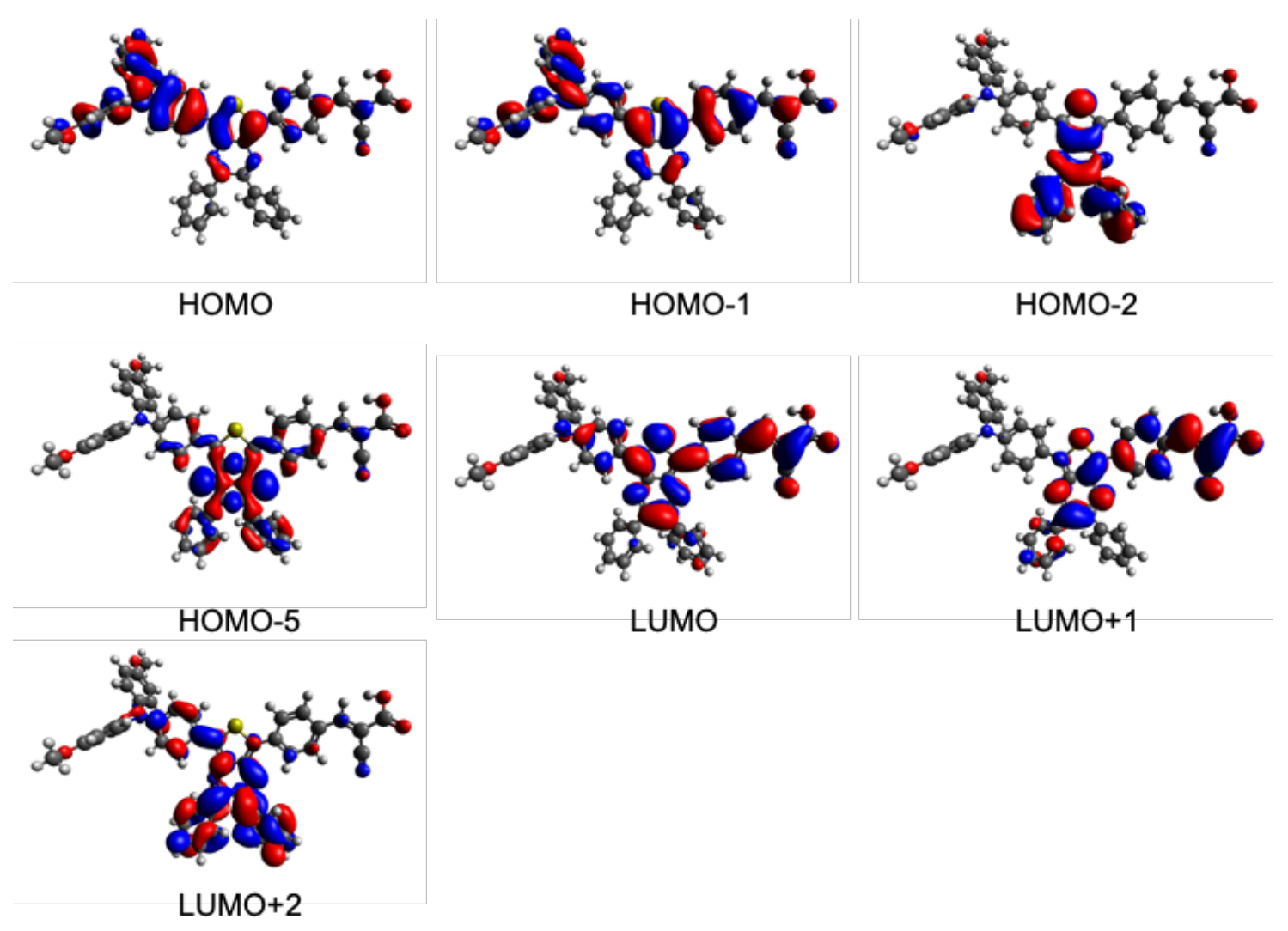

Figure S14. Orbitals for NL6 (in isolation).
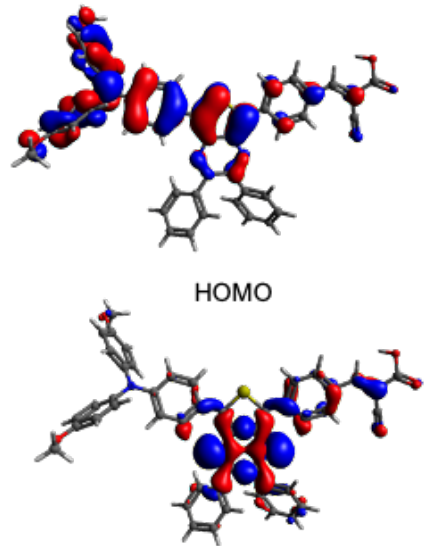

HOMO-5
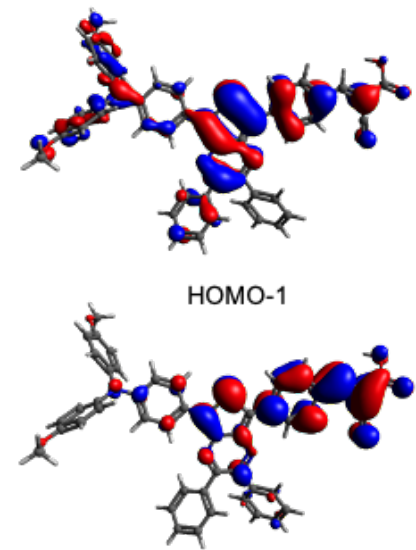

LUMO

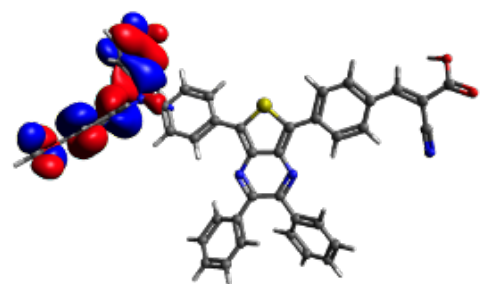

HOMO-3

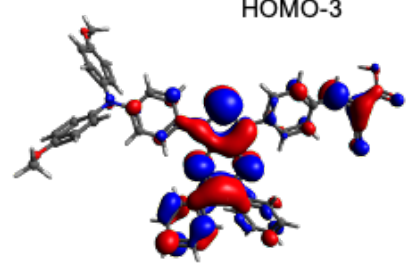

$\mathrm{LUMO}+1$

Figure S15. Orbitals for NL6 (with DCM as implicit solvent). 


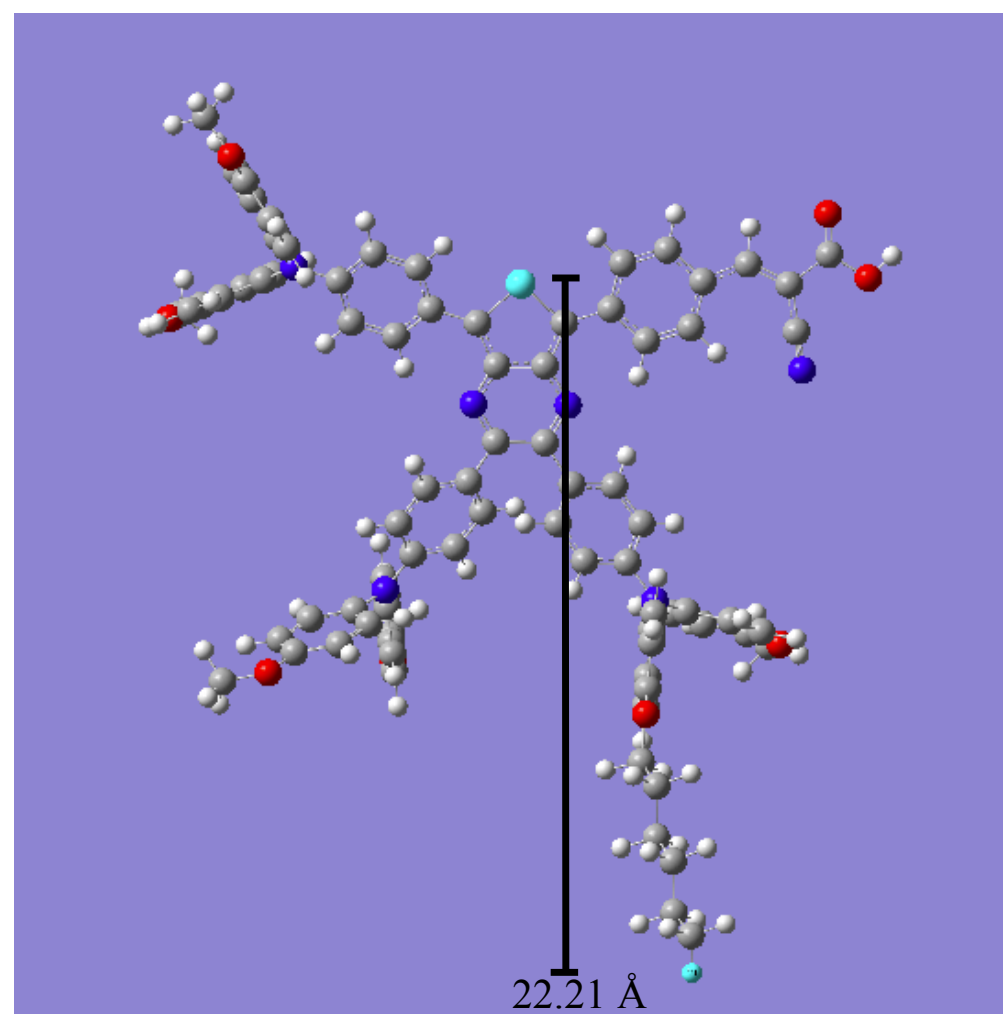

Figure S16. Atomic distance (in angstroms) between the thienopyrazine $\mathrm{S}$ atom and the terminal methyl $\mathrm{H}$ atom as highlighted for JW1.

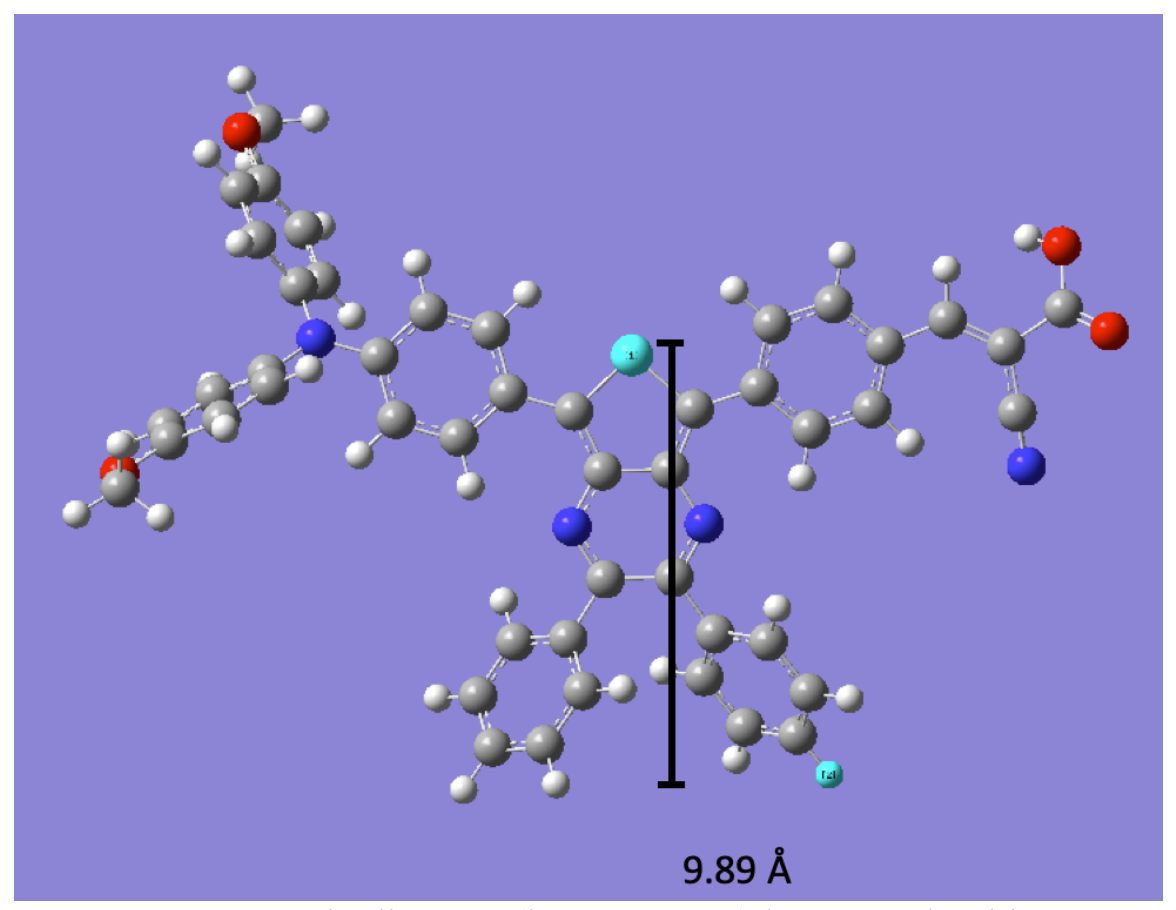

Figure S17. Atomic distance (in angstroms) between the thienopyrazine S atom and the phenyl $\mathrm{H}$ atom as highlighted for NL6. 
5. Computational $X Y Z$ coordinates

(JW1) B3LYP, 6-311G(d,p), in isolation Coordinates

(Angstroms)

$X$ $Y \quad Z$

N

$$
-7.91
$$

1.25397

Z

C

$-6.5744$

1.6831

0.14277

C

$-6.22472$

3.02008

0.07196

C

$-4.90576$

3.43267

0.3369

C

$-3.86476$

2.54781

0.27183

$-4.22325$

1.20945

$-0.07062$

$-5.53984$

0.78937

$-0.33316$

C

C

$-8.38023$

0.19008

$-0.26159$

$-8.13977$

0.18677

$-0.68433$

$-8.60381$

$-0.84937$

$-2.05867$

C

$-9.34297$

$-1.89383$

$-2.86856$

$-9.59793$

$-1.88973$

$-2.30778$

$-9.11611$

$-0.86761$

$-0.93046$

$-8.83996$

1.89609

$-0.13017$

C

C

$$
-8.5301
$$

2.10424

1.01411

$-9.43808$

2.71609

2.36706

C

C

C

$-10.69333$

3.11921

3.21429

$-11.01655$

2.90466

2.73983

$-10.08823$

2.30635

1.39817

$-6.99521$

3.73398

0.54633

$-4.68016$

4.46896

0.59767

$-2.48484$

2.99987

0.4983

$-3.44747$

0.49909

$-0.16254$

$-5.77688$

$-0.24727$

$-0.58015$

$-7.5778$

0.99955

$-0.46449$

$-8.39428-0.81968$

$-2.50304$

$-9.85419$

$-2.94925$

$-3.9293$

$-10.16795$

$-2.709$

$-3.0045$

$-9.31181$

$-0.87815$

$-0.50939$

$-7.56841 \quad 1.78312$

0.93537

$-9.20534$

2.87949

2.74904

$-11.51758$

3.69988

4.25956

3.65796

$\begin{array}{ll}-11.97653 & 3.20457\end{array}$

1

$\begin{array}{lll}-10.3438 & 2.14994 & -0.49482\end{array}$

$-1.28291$

2.27562

$-0.14562$ 


\begin{tabular}{|c|c|c|c|}
\hline C & -0.10629 & 3.09477 & -0.25446 \\
\hline C & -0.37781 & 4.4655 & -0.37813 \\
\hline$S$ & -2.11208 & 4.68652 & -0.3483 \\
\hline C & 0.51025 & 5.60373 & -0.50406 \\
\hline C & 0.00905 & 6.90171 & -0.75236 \\
\hline C & 0.8553 & 7.98416 & -0.86752 \\
\hline C & 2.2563 & 7.8476 & -0.74714 \\
\hline C & 2.75788 & 6.55073 & -0.49687 \\
\hline C & 1.91357 & 5.46465 & -0.37882 \\
\hline C & 3.04922 & 9.04171 & -0.88929 \\
\hline C & 4.3953 & 9.26386 & -0.84983 \\
\hline C & 4.85372 & 10.6668 & -1.04159 \\
\hline $\mathrm{O}$ & 4.12716 & 11.61502 & -1.22771 \\
\hline 0 & 6.19858 & 10.77792 & -0.98547 \\
\hline$N$ & -1.18082 & 0.92412 & -0.07711 \\
\hline$N$ & 1.12737 & 2.53133 & -0.17791 \\
\hline $\mathrm{H}$ & -1.0574 & 7.05996 & -0.86345 \\
\hline $\mathrm{H}$ & 0.43796 & 8.96678 & -1.06045 \\
\hline $\mathrm{H}$ & 3.82142 & 6.39027 & -0.38894 \\
\hline $\mathrm{H}$ & 2.32105 & 4.48416 & -0.18146 \\
\hline $\mathrm{H}$ & 2.48052 & 9.9515 & -1.06369 \\
\hline C & 5.38049 & 8.25847 & -0.64392 \\
\hline $\mathrm{H}$ & 6.40153 & 11.71506 & -1.11837 \\
\hline$N$ & 6.17837 & 7.43757 & -0.47774 \\
\hline C & 0.01781 & 0.38212 & -0.06722 \\
\hline C & 1.21837 & 1.22456 & -0.04397 \\
\hline C & 0.05663 & -1.0994 & -0.12609 \\
\hline C & 0.95654 & -1.79542 & -0.94594 \\
\hline C & 2.86374 & -0.29882 & 1.12165 \\
\hline C & 2.58458 & 0.69389 & 0.17099 \\
\hline C & 3.67014 & 1.26021 & -0.51505 \\
\hline C & 4.97047 & 0.84383 & -0.2838 \\
\hline C & 5.2461 & -0.15258 & 0.67138 \\
\hline C & 4.16271 & -0.71157 & 1.37349 \\
\hline C & 0.91923 & -3.17746 & -1.04812 \\
\hline C & -0.02273 & -3.92877 & -0.32317 \\
\hline C & -0.93229 & -3.23419 & 0.49441 \\
\hline C & -0.89341 & -1.85134 & 0.58037 \\
\hline $\mathrm{H}$ & 3.4815 & 2.04718 & -1.23493 \\
\hline $\mathrm{H}$ & 5.78558 & 1.29918 & -0.83112 \\
\hline
\end{tabular}




\begin{tabular}{|c|c|c|c|}
\hline $\mathrm{H}$ & 4.34565 & -1.47523 & 2.11802 \\
\hline $\mathrm{H}$ & 1.62647 & -3.68475 & -1.69136 \\
\hline $\mathrm{H}$ & -1.6752 & -3.78583 & 1.05615 \\
\hline $\mathrm{H}$ & -1.61637 & -1.33263 & 1.19802 \\
\hline C & -12.8043 & 4.12765 & 3.23589 \\
\hline C & -9.63595 & -3.00258 & -4.40661 \\
\hline $\mathrm{H}$ & -13.28107 & 4.55152 & 4.11812 \\
\hline $\mathrm{H}$ & -12.73779 & 4.89587 & 2.45716 \\
\hline $\mathrm{H}$ & -13.40627 & 3.28911 & 2.86763 \\
\hline $\mathrm{H}$ & -10.08023 & -2.14007 & -4.91624 \\
\hline $\mathrm{H}$ & -8.5678 & -3.05471 & -4.64633 \\
\hline $\mathrm{H}$ & -10.12527 & -3.91277 & -4.74945 \\
\hline $\mathrm{N}$ & 6.56332 & -0.57097 & 0.92001 \\
\hline C & 7.32159 & -1.10044 & -1.35631 \\
\hline C & 7.55365 & -0.52318 & -0.10781 \\
\hline C & 8.79232 & 0.09073 & 0.12743 \\
\hline C & 9.7651 & 0.12067 & -0.8575 \\
\hline C & 9.52105 & -0.44792 & -2.11409 \\
\hline C & 8.28809 & -1.05672 & -2.3609 \\
\hline $\mathrm{H}$ & 8.07082 & -1.50985 & -3.31871 \\
\hline $\mathrm{H}$ & 8.98667 & 0.54382 & 1.09214 \\
\hline $\mathrm{H}$ & 6.37186 & -1.58465 & -1.55053 \\
\hline 0 & 10.53957 & -0.35597 & -3.01643 \\
\hline $\mathrm{H}$ & 10.72318 & 0.59513 & -0.68353 \\
\hline C & 7.63603 & -2.2727 & 2.32272 \\
\hline C & 6.94404 & -1.068 & 2.20292 \\
\hline C & 6.65586 & -0.34112 & 3.36828 \\
\hline C & 7.03657 & -0.81806 & 4.61097 \\
\hline C & 7.73631 & -2.02662 & 4.72413 \\
\hline C & 8.04114 & -2.75022 & 3.5692 \\
\hline $\mathrm{H}$ & 6.12841 & 0.60213 & 3.29087 \\
\hline $\mathrm{H}$ & 7.86971 & -2.84216 & 1.43114 \\
\hline 0 & 8.07339 & -2.40207 & 5.99154 \\
\hline $\mathrm{H}$ & 6.81825 & -0.26197 & 5.51458 \\
\hline C & 10.34541 & -0.90155 & -4.31277 \\
\hline $\mathrm{H}$ & 11.26756 & -0.70787 & -4.85825 \\
\hline $\mathrm{H}$ & 10.17005 & -1.98276 & -4.27274 \\
\hline $\mathrm{H}$ & 9.51027 & -0.41763 & -4.83185 \\
\hline$N$ & -0.05477 & -5.33253 & -0.41358 \\
\hline C & 1.27914 & -7.05216 & -1.55267 \\
\hline
\end{tabular}




\begin{tabular}{|c|c|c|c|}
\hline C & 0.3573 & -5.99634 & -1.6076 \\
\hline C & -0.1548 & -5.62384 & -2.85092 \\
\hline C & 0.24986 & -6.26855 & -4.0197 \\
\hline C & 1.165 & -7.32193 & -3.95275 \\
\hline C & 1.67035 & -7.71144 & -2.70597 \\
\hline $\mathrm{H}$ & 2.38126 & -8.52804 & -2.67023 \\
\hline $\mathrm{H}$ & -0.87287 & -4.81455 & -2.90964 \\
\hline $\mathrm{H}$ & 1.68417 & -7.35475 & -0.59444 \\
\hline $\mathrm{O}$ & 1.62348 & -8.02686 & -5.02726 \\
\hline $\mathrm{H}$ & -0.16547 & -5.94892 & -4.96598 \\
\hline C & -1.41964 & -7.15183 & 0.50217 \\
\hline C & -0.49173 & -6.12707 & 0.68782 \\
\hline C & 0.02181 & -5.91516 & 1.9767 \\
\hline C & -0.39447 & -6.69571 & 3.04174 \\
\hline C & -1.3215 & -7.72786 & 2.84683 \\
\hline C & -1.82815 & -7.95658 & 1.56576 \\
\hline $\mathrm{H}$ & -2.54549 & -8.74492 & 1.38149 \\
\hline $\mathrm{H}$ & 0.74958 & -5.12893 & 2.1376 \\
\hline $\mathrm{H}$ & -1.82317 & -7.33059 & -0.48729 \\
\hline $\mathrm{O}$ & -1.65647 & -8.44456 & 3.95822 \\
\hline $\mathrm{H}$ & -0.00263 & -6.53613 & 4.03897 \\
\hline C & 1.15037 & -7.67413 & -6.31862 \\
\hline C & -2.59756 & -9.49914 & 3.82296 \\
\hline $\mathrm{H}$ & 1.64712 & -8.3503 & -7.01253 \\
\hline $\mathrm{H}$ & 0.0655 & -7.8064 & -6.4007 \\
\hline $\mathrm{H}$ & 1.40961 & -6.64027 & -6.57385 \\
\hline $\mathrm{H}$ & -2.23619 & -10.27625 & 3.13972 \\
\hline $\mathrm{H}$ & -3.56784 & -9.13012 & 3.47157 \\
\hline $\mathrm{H}$ & -2.71441 & -9.92328 & 4.8189 \\
\hline $\mathrm{H}$ & 8.58122 & -3.68578 & 3.62283 \\
\hline C & 8.78584 & -3.61734 & 6.16891 \\
\hline $\mathrm{H}$ & 8.94659 & -3.71423 & 7.24139 \\
\hline $\mathrm{H}$ & 8.20917 & -4.47795 & 5.81109 \\
\hline $\mathrm{H}$ & 9.75573 & -3.59359 & 5.65908 \\
\hline $\mathrm{H}$ & 1.6979 & -1.25097 & -1.51737 \\
\hline $\mathrm{H}$ & 2.05611 & -0.75218 & 1.68258 \\
\hline
\end{tabular}


(JW1) B3LYP, 6-311G(d,p), DCM Coordinates

(Angstroms)

$X$

$Y$

Z

$\mathrm{N}$

$-7.918484$

1.192776

0.086202

C

$-6.59309$

1.635222

0.039786

C

$-6.253048$

2.961289

0.37704

$-4.938968$

3.389952

0.33333

$-3.887672$

2.533407

$-0.052347$

$-4.234972$

1.207375

$-0.386606$

$-5.546923$

0.770794

$-0.342111$

$-8.358394$

0.098926

$-0.72415$

C

$-8.169402$

0.108744

$-2.106435$

C

$-8.605792$

$-0.95176$

$-2.899884$

C

$-9.263577$

$-2.03617$

$-2.311088$

$-9.466762$

$-2.044653$

$-0.924078$

$-9.01325$

$-0.994787$

$-0.141499$

$-8.880736$

1.82746

0.932452

$-8.630822$

1.996756

2.302309

$-9.57233$

2.597054

3.122263

$-10.80037$

3.026626

2.599285

$-11.06307$

2.850038

1.237818

$-10.10148$

2.261689

0.416269

$-7.0277$

3.654979

0.675673

$-4.725351$

4.415537

0.613452

$-2.513973$

3.003818

$-0.121758$

$-3.454963$

0.51664

$-0.672592$

$-5.770605$

$-0.255193$

$-0.603651$

$-7.673313$

0.951914

$-2.572416$

$-8.440386$

$-0.911546$

$-3.967764$

$-9.739751$

$-3.11706$

$-2.989896$

$-9.974632$

$-2.891848$

$-0.478929$

$-9.168305$

$-1.015599$

0.930649

$-7.692448$

1.654332

2.722052

$-9.384368$

2.728953

4.181107

$-11.66154$

3.592465

3.490364

$-12.00004$

3.170833

0.803488

$-10.30999$

2.135142

$-0.639396$

$-1.302693$

2.291623

$-0.11116$

$-0.135943$

3.124717

$-0.204223$ 


\begin{tabular}{|c|c|c|c|}
\hline C & -0.423902 & 4.496325 & -0.30069 \\
\hline$S$ & -2.162842 & 4.697343 & -0.277007 \\
\hline C & 0.448644 & 5.646159 & -0.394996 \\
\hline C & -0.069983 & 6.962226 & -0.438028 \\
\hline C & 0.763037 & 8.055848 & -0.535809 \\
\hline C & 2.169319 & 7.916148 & -0.596256 \\
\hline C & 2.688856 & 6.601691 & -0.546343 \\
\hline C & 1.858387 & 5.504334 & -0.449544 \\
\hline C & 2.944853 & 9.121275 & -0.700957 \\
\hline C & 4.289938 & 9.351747 & -0.795075 \\
\hline C & 4.737518 & 10.766389 & -0.889067 \\
\hline $\mathrm{O}$ & 3.999907 & 11.727192 & -0.887791 \\
\hline $\mathrm{O}$ & 6.077663 & 10.871617 & -0.97793 \\
\hline$N$ & -1.188226 & 0.940856 & -0.051835 \\
\hline$N$ & 1.103865 & 2.573213 & -0.128411 \\
\hline $\mathrm{H}$ & -1.139731 & 7.127989 & -0.392153 \\
\hline $\mathrm{H}$ & 0.331507 & 9.05017 & -0.567051 \\
\hline H & 3.756269 & 6.43558 & -0.582553 \\
\hline H & 2.28052 & 4.511469 & -0.405426 \\
\hline $\mathrm{H}$ & 2.361513 & 10.038014 & -0.710058 \\
\hline C & 5.289302 & 8.341678 & -0.812984 \\
\hline $\mathrm{H}$ & 6.293233 & 11.814982 & -1.03476 \\
\hline$N$ & 6.109742 & 7.52535 & -0.829202 \\
\hline C & 0.016463 & 0.410091 & -0.041268 \\
\hline C & 1.207222 & 1.264492 & -0.011334 \\
\hline C & 0.074246 & -1.070054 & -0.099023 \\
\hline C & 0.965627 & -1.754768 & -0.938504 \\
\hline C & 2.867922 & -0.245539 & 1.147948 \\
\hline C & 2.576898 & 0.740217 & 0.1927 \\
\hline C & 3.654741 & 1.29799 & -0.512879 \\
\hline C & 4.957385 & 0.879096 & -0.297779 \\
\hline C & 5.246334 & -0.111142 & 0.662287 \\
\hline C & 4.169314 & -0.658625 & 1.386498 \\
\hline C & 0.945325 & -3.137326 & -1.0402 \\
\hline C & 0.031835 & -3.904225 & -0.291628 \\
\hline C & -0.867644 & -3.219387 & 0.547962 \\
\hline C & -0.847471 & -1.83582 & 0.630504 \\
\hline $\mathrm{H}$ & 3.460568 & 2.074517 & -1.242815 \\
\hline $\mathrm{H}$ & 5.763002 & 1.325303 & -0.865945 \\
\hline $\mathrm{H}$ & 4.357372 & -1.415874 & 2.136017 \\
\hline
\end{tabular}




\begin{tabular}{|c|c|c|c|}
\hline $\mathrm{H}$ & 1.64421 & -3.631647 & -1.702107 \\
\hline $\mathrm{H}$ & -1.588759 & -3.778072 & 1.130306 \\
\hline $\mathrm{H}$ & -1.56157 & -1.330805 & 1.269623 \\
\hline C & -12.93092 & 4.04167 & 3.017982 \\
\hline C & -9.572434 & -3.158397 & -4.406503 \\
\hline $\mathrm{H}$ & -13.44379 & 4.447309 & 3.887773 \\
\hline $\mathrm{H}$ & -12.82043 & 4.826486 & 2.26313 \\
\hline $\mathrm{H}$ & -13.51690 & 3.215191 & 2.603688 \\
\hline $\mathrm{H}$ & -10.08539 & -2.322551 & -4.892484 \\
\hline $\mathrm{H}$ & -8.513507 & -3.148822 & -4.683201 \\
\hline $\mathrm{H}$ & -10.02169 & -4.095113 & -4.730315 \\
\hline$N$ & 6.561795 & -0.533195 & 0.891955 \\
\hline C & 7.34243 & -1.016799 & -1.38928 \\
\hline C & 7.554447 & -0.446194 & -0.133722 \\
\hline C & 8.77562 & 0.195484 & 0.115374 \\
\hline C & 9.753471 & 0.258797 & -0.864627 \\
\hline C & 9.530173 & -0.304397 & -2.128511 \\
\hline C & 8.312731 & -0.941066 & -2.388226 \\
\hline $\mathrm{H}$ & 8.112392 & -1.391577 & -3.350696 \\
\hline $\mathrm{H}$ & 8.955813 & 0.642947 & 1.085585 \\
\hline $\mathrm{H}$ & 6.407438 & -1.524698 & -1.594382 \\
\hline 0 & 10.549508 & -0.181781 & -3.024697 \\
\hline $\mathrm{H}$ & 10.698974 & 0.753179 & -0.676158 \\
\hline C & 7.583865 & -2.324639 & 2.221376 \\
\hline C & 6.948279 & -1.085097 & 2.153263 \\
\hline C & 6.723381 & -0.37838 & 3.343852 \\
\hline C & 7.112123 & -0.908006 & 4.563608 \\
\hline C & 7.756265 & -2.151898 & 4.625481 \\
\hline C & 7.996333 & -2.857264 & 3.443041 \\
\hline $\mathrm{H}$ & 6.240469 & 0.590975 & 3.307803 \\
\hline $\mathrm{H}$ & 7.766052 & -2.881612 & 1.309977 \\
\hline $\mathrm{O}$ & 8.107845 & -2.579487 & 5.870751 \\
\hline $\mathrm{H}$ & 6.940961 & -0.365513 & 5.485744 \\
\hline C & 10.38015 & -0.740219 & -4.326629 \\
\hline $\mathrm{H}$ & 11.302588 & -0.526507 & -4.862939 \\
\hline $\mathrm{H}$ & 10.230374 & -1.823458 & -4.27906 \\
\hline $\mathrm{H}$ & 9.539006 & -0.277423 & -4.852385 \\
\hline$N$ & 0.017488 & -5.304154 & -0.379747 \\
\hline C & 1.434157 & -6.981917 & -1.481766 \\
\hline C & 0.466539 & -5.970444 & -1.561815 \\
\hline
\end{tabular}




$\begin{array}{lrrr}\mathrm{C} & -0.059103 & -5.646192 & -2.813219 \\ \mathrm{C} & 0.375888 & -6.295828 & -3.968261 \\ \mathrm{C} & 1.337893 & -7.30628 & -3.87757 \\ \mathrm{C} & 1.857786 & -7.647037 & -2.621426 \\ \mathrm{H} & 2.604395 & -8.430145 & -2.563802 \\ \mathrm{H} & -0.814909 & -4.873638 & -2.891275 \\ \mathrm{H} & 1.851365 & -7.246881 & -0.51753 \\ \mathrm{O} & 1.827893 & -8.010689 & -4.936665 \\ \mathrm{H} & -0.051633 & -6.015883 & -4.921308 \\ \mathrm{C} & -1.394 & -7.103901 & 0.506524 \\ \mathrm{C} & -0.441324 & -6.10556 & 0.710674 \\ \mathrm{C} & 0.077268 & -5.926944 & 2.001985 \\ \mathrm{C} & -0.359174 & -6.715468 & 3.054203 \\ \mathrm{C} & -1.312203 & -7.721523 & 2.841494 \\ \mathrm{C} & -1.824421 & -7.916334 & 1.555899 \\ \mathrm{H} & -2.560984 & -8.683135 & 1.358294 \\ \mathrm{H} & 0.825575 & -5.163295 & 2.177514 \\ \mathrm{H} & -1.802618 & -7.256189 & -0.485396 \\ \mathrm{O} & -1.666244 & -8.448833 & 3.938697 \\ \mathrm{H} & 0.038641 & -6.58007 & 4.052907 \\ \mathrm{C} & 1.337694 & -7.705878 & -6.241371 \\ \mathrm{C} & -2.63643 & -9.48318 & 3.78315 \\ \mathrm{H} & 1.866923 & -8.37224 & -6.919694 \\ \mathrm{H} & 0.26164 & -7.891594 & -6.317031 \\ \mathrm{H} & 1.549514 & -6.667163 & -6.514207 \\ \mathrm{H} & -2.290406 & -10.25300 & 3.086123 \\ \mathrm{H} & -3.594762 & -9.08197 & 3.438579 \\ \mathrm{H} & -2.763081 & -9.921619 & 4.771069 \\ \mathrm{H} & 8.490304 & -3.819166 & 3.457184 \\ \mathrm{H} & 8.768446 & -3.838053 & 5.995227 \\ \mathrm{H} & & -3.971371 & 7.05922 \\ \mathrm{H} & -668133 & -0.691863 & 1.725585\end{array}$


(NL6) B3LYP, 6-311G(d,p), in isolation Coordinates

(Angstroms)

$X$

$Y$

Z

C

4.627681

5.351955

2.42575

C

C

$-0.987249$

5.714674

1.449481

$0.13864-0.458345$

$-0.127035$

C

0.938927

0.676349

0.092016

2.351883

0.414401

0.037582

2.673231

$-0.920727$

$-0.243963$

5.304803

$-4.575827$

$-1.290892$

$-0.88325$

7.778564

0.842729

2.780553

2.613331

0.554965

1.347942

2.898911

0.502316

$\begin{array}{ll}-7.818332 & -5.716884\end{array}$

3.694934

$-8.702755$

3.045654

$-4.023769$

$3.95647-1.581112$

$-0.382311$

5.167043

$-0.913894$

$-0.080151$

$\begin{array}{ll}7.282755 & -0.986919\end{array}$

0.033474

4.049656

$-2.92087$

$-0.82043$

5.124461

0.108648

0.26448

$-1.306451$

$-0.610773$

$-0.135659$

$-2.167618$

0.499916

$-0.256429$

$-3.542324$

0.347766

$-0.271701$

$-4.138838 \quad-0.923775$

$-0.169648$

$-3.28507 \quad-2.038415$

$-0.05225$

$-1.912364-1.879233$

$-0.032083$

$-1.74052$

1.489997

$-0.323802$

$-4.170418$

1.224744

$-0.359458$

$5.272118-3.546765$

$-0.947681$

$-3.708453-3.031206$

0.025455

$-1.295712$

$-2.765273$

0.072267

$-0.423277$

6.798893

0.778966

0.732469

6.616484

0.02242

6.48182

$-2.880121$

$-0.655284$

6.608416

6.090009

2.018322

6.389468

$-1.542574$

$-0.213676$

$-6.367414$

$-0.124564$

$-0.843333$

$\begin{array}{ll}-7.455097 & 0.451687\end{array}$

$-0.172218$

$-8.281334$

1.358746

$-0.814091$ 


\begin{tabular}{|c|c|c|c|}
\hline C & -8.033862 & 1.728252 & -2.142459 \\
\hline C & -6.94932 & 1.162492 & -2.818473 \\
\hline C & -6.134146 & 0.235204 & -2.170747 \\
\hline $\mathrm{H}$ & -7.650795 & 0.177783 & 0.857569 \\
\hline $\mathrm{H}$ & -9.124375 & 1.807342 & -0.302901 \\
\hline C & 7.708406 & -3.625693 & -0.826879 \\
\hline $\mathrm{H}$ & -6.735947 & 1.420596 & -3.846922 \\
\hline $\mathrm{H}$ & -5.302142 & -0.208133 & -2.704846 \\
\hline C & -6.159336 & -2.170758 & 0.487001 \\
\hline C & -7.124377 & -2.945565 & -0.17248 \\
\hline C & -7.757737 & -3.9904 & 0.479088 \\
\hline C & -7.432105 & -4.30233 & 1.805304 \\
\hline C & -6.467982 & -3.539369 & 2.469586 \\
\hline C & -5.849901 & -2.475966 & 1.812822 \\
\hline $\mathrm{H}$ & -7.378801 & -2.717152 & -1.200496 \\
\hline $\mathrm{H}$ & -8.50544 & -4.592141 & -0.023038 \\
\hline $\mathrm{H}$ & -6.200394 & -3.748593 & 3.496491 \\
\hline $\mathrm{H}$ & -5.114291 & -1.879745 & 2.339485 \\
\hline C & 9.021707 & -3.273338 & -0.718622 \\
\hline $\mathrm{H}$ & 2.694148 & 4.44267 & 2.598473 \\
\hline $\mathrm{H}$ & 5.174671 & 2.994656 & -0.588586 \\
\hline $\mathrm{H}$ & 7.5354 & -4.654035 & -1.136408 \\
\hline $\mathrm{H}$ & 4.475484 & 5.996586 & 3.283888 \\
\hline $\mathrm{H}$ & 6.953708 & 4.583852 & 0.075381 \\
\hline C & 1.332088 & 5.363038 & -0.051957 \\
\hline $\mathrm{H}$ & -0.841365 & 3.608155 & 1.869503 \\
\hline $\mathrm{H}$ & -1.887959 & 5.847873 & 2.03818 \\
\hline C & 0.774637 & 4.26773 & 0.620839 \\
\hline C & 10.128744 & -4.242585 & -1.025048 \\
\hline $\mathrm{H}$ & 1.169742 & 7.451657 & -0.512818 \\
\hline $\mathrm{H}$ & 2.228876 & 5.234609 & -0.645166 \\
\hline$S$ & 1.182145 & -1.813711 & -0.42825 \\
\hline $\mathrm{N}$ & 3.240838 & 1.409895 & 0.293588 \\
\hline$N$ & 0.477633 & 1.93234 & 0.309912 \\
\hline $\mathrm{N}$ & -5.531225 & -1.076554 & -0.182588 \\
\hline C & -0.399111 & 4.45736 & 1.363212 \\
\hline C & 3.805276 & 3.621058 & 0.942221 \\
\hline C & 3.620062 & 4.474563 & 2.037833 \\
\hline C & 5.022367 & 3.664785 & 0.248443 \\
\hline C & 5.827205 & 5.40085 & 1.718305 \\
\hline
\end{tabular}




$\begin{array}{lrrr}\mathrm{C} & 6.021197 & 4.555136 & 0.627376 \\ \mathrm{H} & -8.458181 & -6.569644 & 3.915136 \\ \mathrm{H} & -6.769761 & -6.01072 & 3.817159 \\ \mathrm{H} & -8.050747 & -4.902062 & 4.38976 \\ \mathrm{H} & -9.494307 & 3.762993 & -4.233428 \\ \mathrm{H} & -7.729769 & 3.531651 & -4.157406 \\ \mathrm{H} & -8.786357 & 2.202369 & -4.718419 \\ \mathrm{O} & -8.104432 & -5.354357 & 2.351404 \\ \mathrm{O} & -8.899311 & 2.63393 & -2.678155 \\ \mathrm{H} & 3.152508 & -3.47414 & -1.0727 \\ \mathrm{O} & 11.209429 & -3.901632 & -1.413093 \\ \mathrm{O} & 9.837609 & -5.563143 & -0.85822 \\ \mathrm{C} & 9.470017 & -1.952333 & -0.429418 \\ \mathrm{~N} & 9.834872 & -0.884578 & -0.178618 \\ \mathrm{H} & 9.046872 & -5.662965 & -0.315987\end{array}$


(NL6) B3LYP, 6-311G(d,p), DCM Coordinates (Angstroms)

$X$ $Y \quad Z$

C $-4.627681$

5.351955

C 0.987249 5.714674 $-2.42575$

C $-0.13864$ $-0.458345$ $-1.449481$

C

C

C

$\mathrm{H}$

$\mathrm{H}$

C

C

C

C

C

C

$\mathrm{H}$

C

$\mathrm{H}$

C

C

C

C

C

C

$\mathrm{H}$

$\mathrm{H}$

C

$\mathrm{H}$

$\mathrm{H}$

C

C

C

$\mathrm{H}$

C

C

C

C

$-0.938927$

0.676349

0.127035

$-2.351883$

0.414401

$-0.092016$

$-2.673231$

$-0.920727$

$-0.037582$

$-5.304803$

$-4.575827$

0.243963

0.88325

7.778564

1.290892

$-2.780553$

2.613331

$-0.842729$

$-1.347942$

2.898911

$-0.554965$

7.818332

$-5.716884$

$-0.502316$

8.702755

3.045654

$-3.694934$

$-3.95647$

$-1.581112$

4.023769

$-5.167043$

$-0.913894$

0.382311

$-7.28275$

$-0.986919$

0.080151

$-4.049656$

$-2.92087$

$-0.033474$

$-5.124461$

0.108648

0.82043

1.306451

$-0.610773$

$-0.26448$

2.167618

0.499916

0.135659

3.542324

0.347766

0.256429

4.138838

$-0.923775$

0.271701

$3.28507 \quad-2.038415$

0.169648

1.912364

$-1.879233$

0.05225

1.74052

1.489997

0.032083

0.323802

4.170418

1.224744

0.359458

$-5.272118$

$-3.546765$

0.947681

$3.708453-3.031206$

$-0.025455$

1.295712

$-2.765273$

$-0.072267$

0.423277

6.798893

$-0.778966$

$-0.732469$

$6.616484-0.02242$

$-6.48182$

$-2.880121$

0.655284

$-6.608416$

6.090009

$-2.018322$

$-6.389468$

$-1.542574$

0.213676

6.367414

$-0.124564$

0.843333

7.455097

0.451687

0.172218

8.281334

1.358746

0.814091 


\begin{tabular}{|c|c|c|c|}
\hline C & 8.033862 & 1.728252 & 2.142459 \\
\hline C & 6.94932 & 1.162492 & 2.818473 \\
\hline C & 6.134146 & 0.235204 & 2.170747 \\
\hline $\mathrm{H}$ & 7.650795 & 0.177783 & -0.857569 \\
\hline $\mathrm{H}$ & 9.124375 & 1.807342 & 0.302901 \\
\hline C & -7.708406 & -3.625693 & 0.826879 \\
\hline $\mathrm{H}$ & 6.735947 & 1.420596 & 3.846922 \\
\hline $\mathrm{H}$ & 5.302142 & -0.208133 & 2.704846 \\
\hline C & 6.159336 & -2.170758 & -0.487001 \\
\hline C & 7.124377 & -2.945565 & 0.17248 \\
\hline C & 7.757737 & -3.9904 & -0.479088 \\
\hline C & 7.432105 & -4.30233 & -1.805304 \\
\hline C & 6.467982 & -3.539369 & -2.469586 \\
\hline C & 5.849901 & -2.475966 & -1.812822 \\
\hline $\mathrm{H}$ & 7.378801 & -2.717152 & 1.200496 \\
\hline $\mathrm{H}$ & 8.50544 & -4.592141 & 0.023038 \\
\hline $\mathrm{H}$ & 6.200394 & -3.748593 & -3.496491 \\
\hline $\mathrm{H}$ & 5.114291 & -1.879745 & -2.339485 \\
\hline C & -9.021707 & -3.273338 & 0.718622 \\
\hline $\mathrm{H}$ & -2.694148 & 4.44267 & -2.598473 \\
\hline $\mathrm{H}$ & -5.174671 & 2.994656 & 0.588586 \\
\hline $\mathrm{H}$ & -7.5354 & -4.654035 & 1.136408 \\
\hline $\mathrm{H}$ & -4.475484 & 5.996586 & -3.283888 \\
\hline $\mathrm{H}$ & -6.953708 & 4.583852 & -0.075381 \\
\hline C & -1.332088 & 5.363038 & 0.051957 \\
\hline $\mathrm{H}$ & 0.841365 & 3.608155 & -1.869503 \\
\hline $\mathrm{H}$ & 1.887959 & 5.847873 & -2.03818 \\
\hline C & -0.774637 & 4.26773 & -0.620839 \\
\hline C & -10.128744 & -4.242585 & 1.025048 \\
\hline $\mathrm{H}$ & -1.169742 & 7.451657 & 0.512818 \\
\hline $\mathrm{H}$ & -2.228876 & 5.234609 & 0.645166 \\
\hline$S$ & -1.182145 & -1.813711 & 0.42825 \\
\hline$N$ & -3.240838 & 1.409895 & -0.293588 \\
\hline$N$ & -0.477633 & 1.93234 & -0.309912 \\
\hline $\mathrm{N}$ & 5.531225 & -1.076554 & 0.182588 \\
\hline C & 0.399111 & 4.45736 & -1.363212 \\
\hline C & -3.805276 & 3.621058 & -0.942221 \\
\hline C & -3.620062 & 4.474563 & -2.037833 \\
\hline C & -5.022367 & 3.664785 & -0.248443 \\
\hline C & -5.827205 & 5.40085 & -1.718305 \\
\hline
\end{tabular}




$\begin{array}{lrrr}\mathrm{C} & -6.021197 & 4.555136 & -0.627376 \\ \mathrm{H} & 8.458181 & -6.569644 & -3.915136 \\ \mathrm{H} & 6.769761 & -6.01072 & -3.817159 \\ \mathrm{H} & 8.050747 & -4.902062 & -4.38976 \\ \mathrm{H} & 9.494307 & 3.762993 & 4.233428 \\ \mathrm{H} & 7.729769 & 3.531651 & 4.157406 \\ \mathrm{H} & 8.786357 & 2.202369 & 4.718419 \\ \mathrm{O} & 8.104432 & -5.354357 & -2.351404 \\ \mathrm{O} & 8.899311 & 2.63393 & 2.678155 \\ \mathrm{H} & -3.152508 & -3.47414 & 1.0727 \\ \mathrm{O} & -11.209429 & -3.901632 & 1.413093 \\ \mathrm{O} & -9.837609 & -5.563143 & 0.85822 \\ \mathrm{C} & -9.470017 & -1.952333 & 0.429418 \\ \mathrm{~N} & -9.834872 & -0.884578 & 0.178618 \\ \mathrm{H} & -9.046872 & -5.662965 & 0.315987\end{array}$




\section{NMR Spectra}

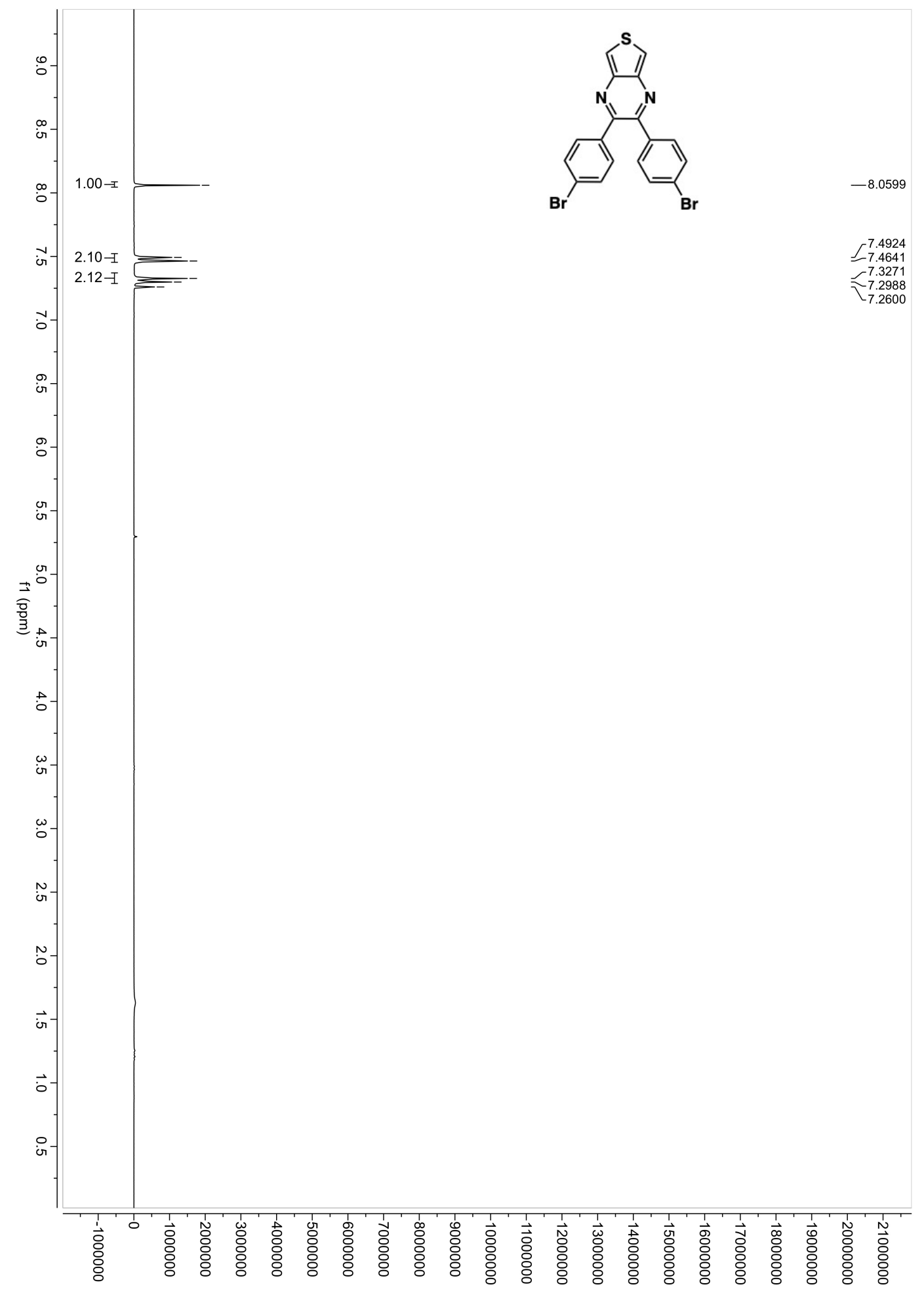

Figure S18. ${ }^{1} \mathrm{H} \mathrm{NMR}\left(\mathrm{CDCl}_{3}\right), 300 \mathrm{MHz}$ (compound 3). 


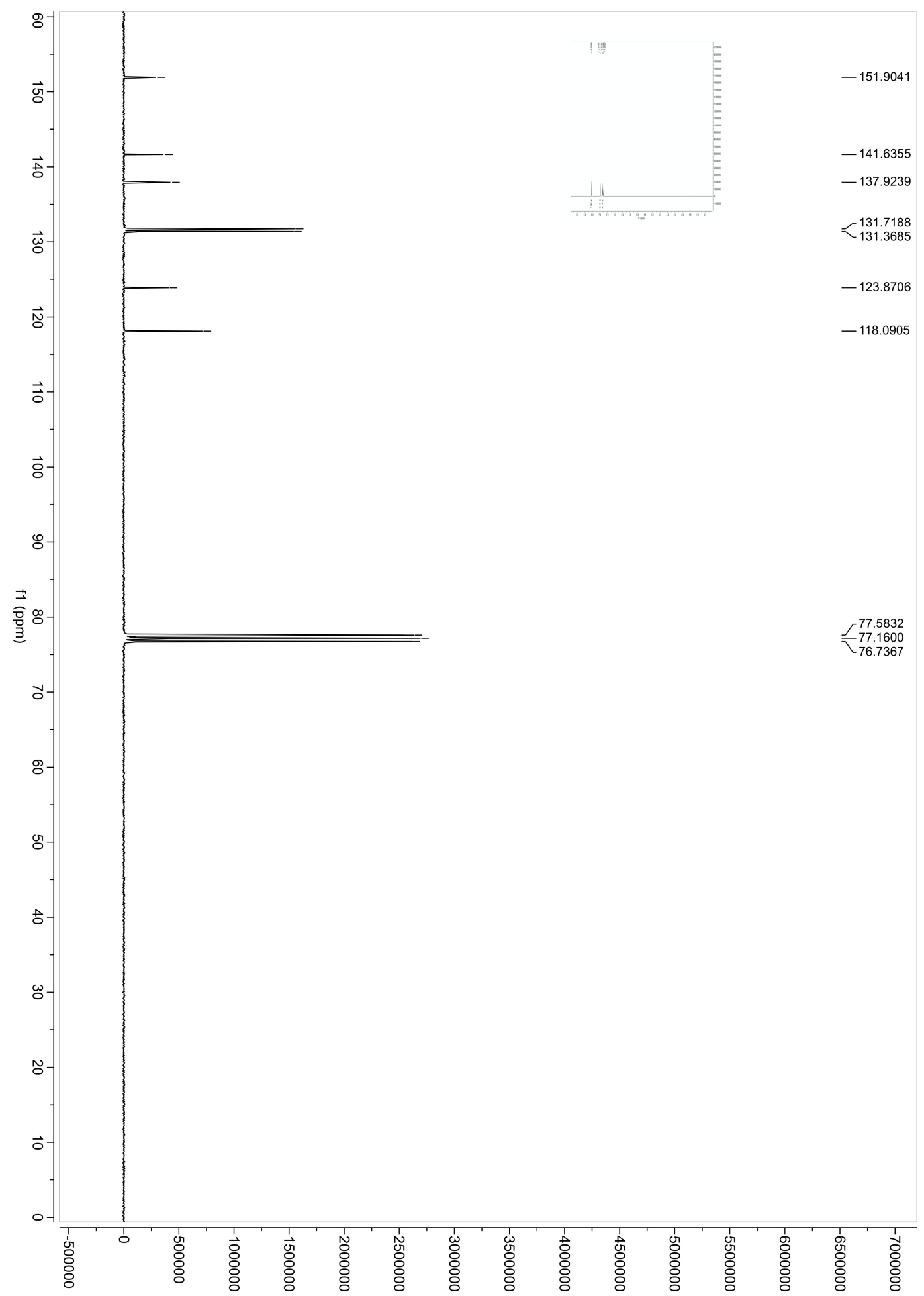

Figure S19. ${ }^{13} \mathrm{C} \mathrm{NMR}\left(\mathrm{CDCl}_{3}\right), 75 \mathrm{MHz}$ (compound 3). 


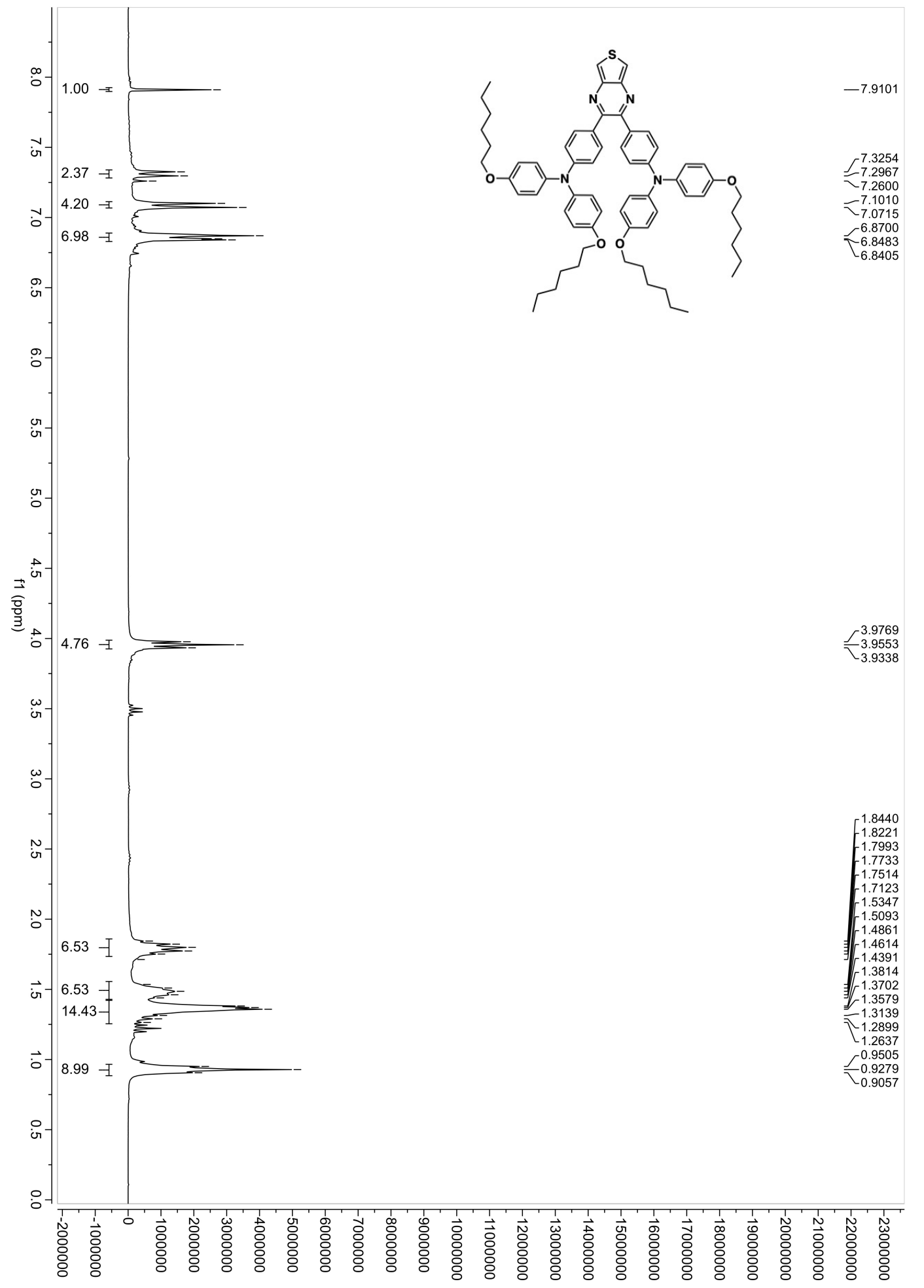

Figure S20. ${ }^{1} \mathrm{H} \mathrm{NMR}\left(\mathrm{CDCl}_{3}\right), 300 \mathrm{MHz}$ (compound 5). 


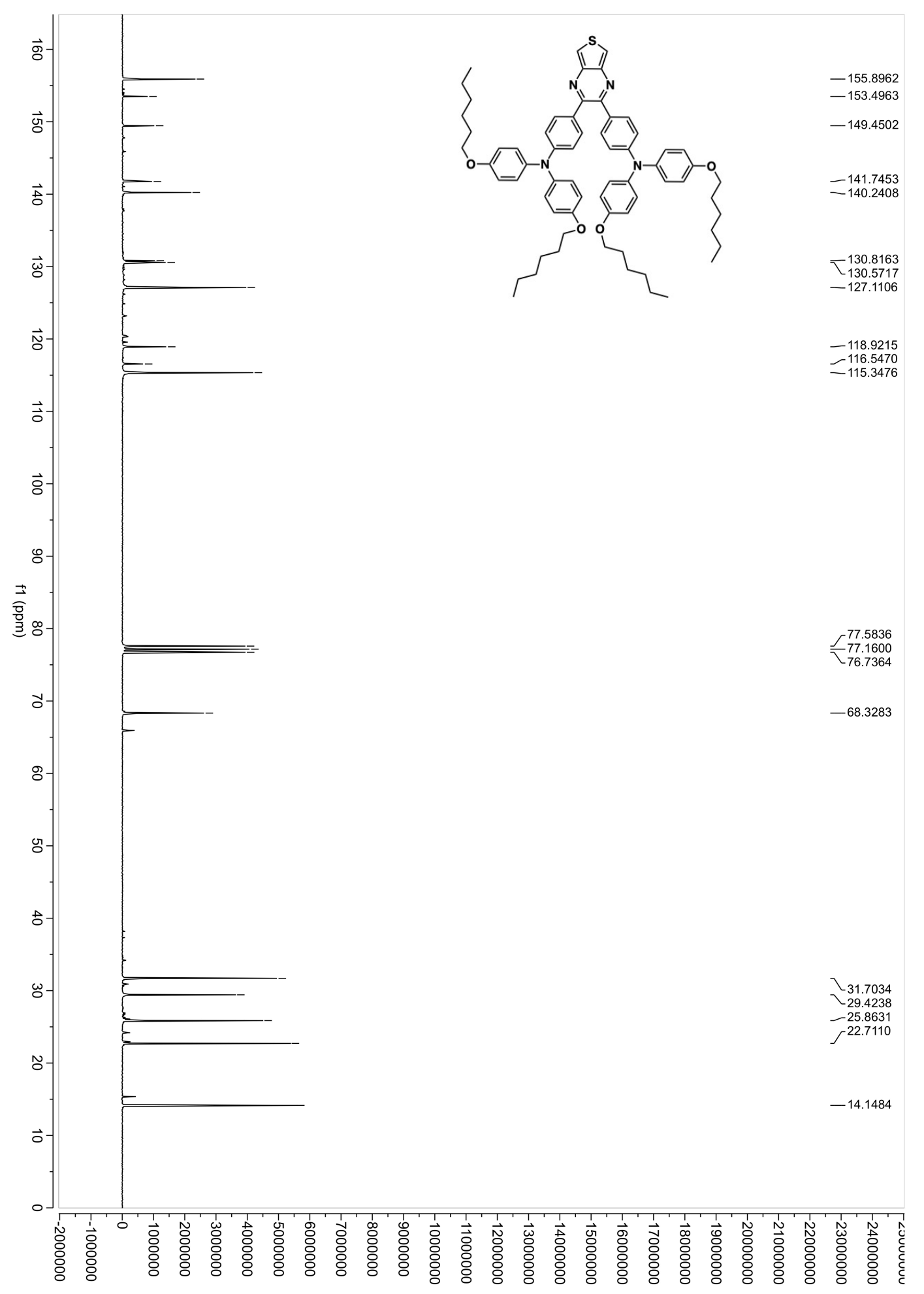

Figure S21. ${ }^{13} \mathrm{C} \mathrm{NMR}\left(\mathrm{CDCl}_{3}\right), 75 \mathrm{MHz}$ (compound 5). 


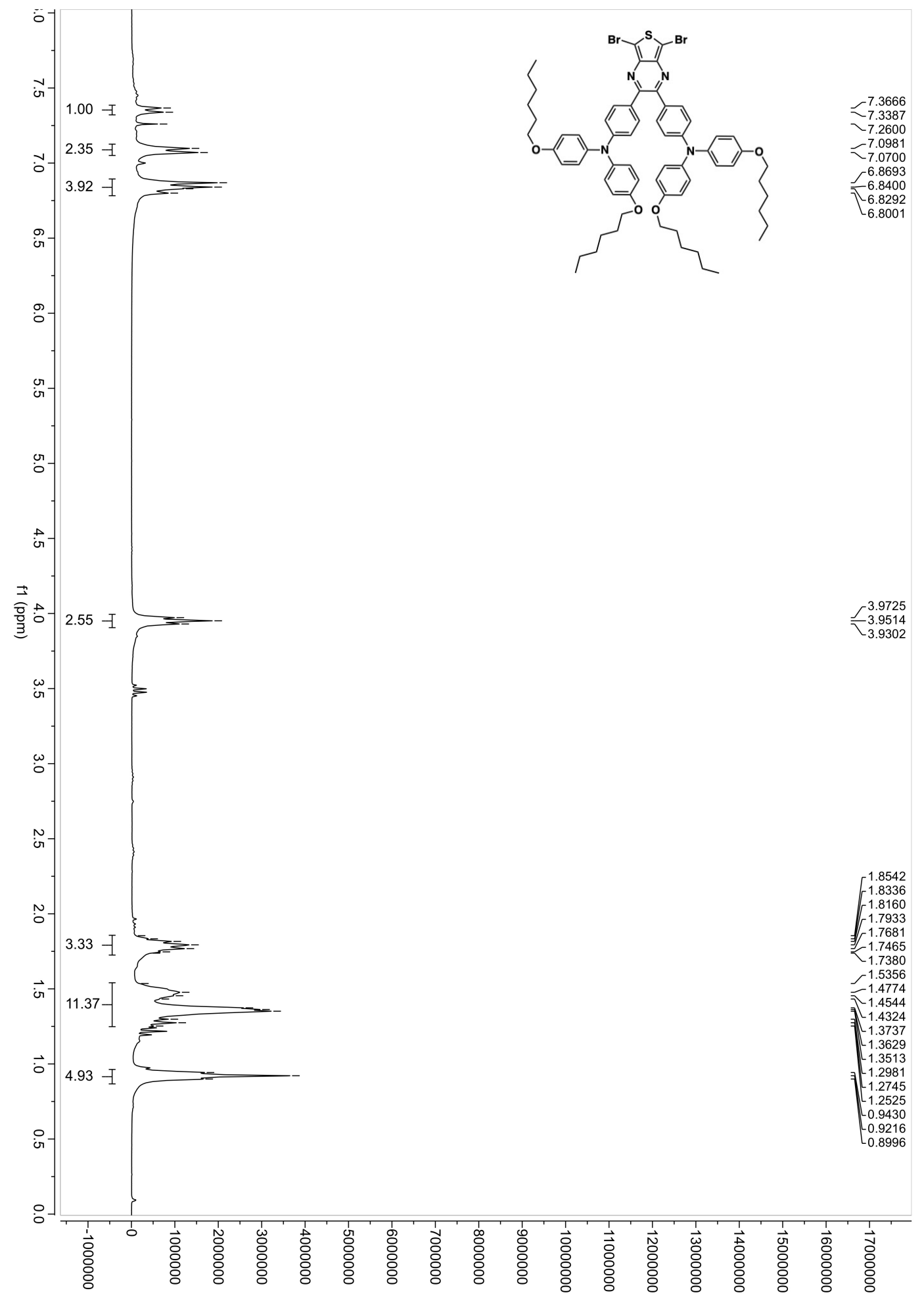

Figure S22. ${ }^{1} \mathrm{H} \mathrm{NMR}\left(\mathrm{CDCl}_{3}\right), 300 \mathrm{MHz}$ (compound 6). 


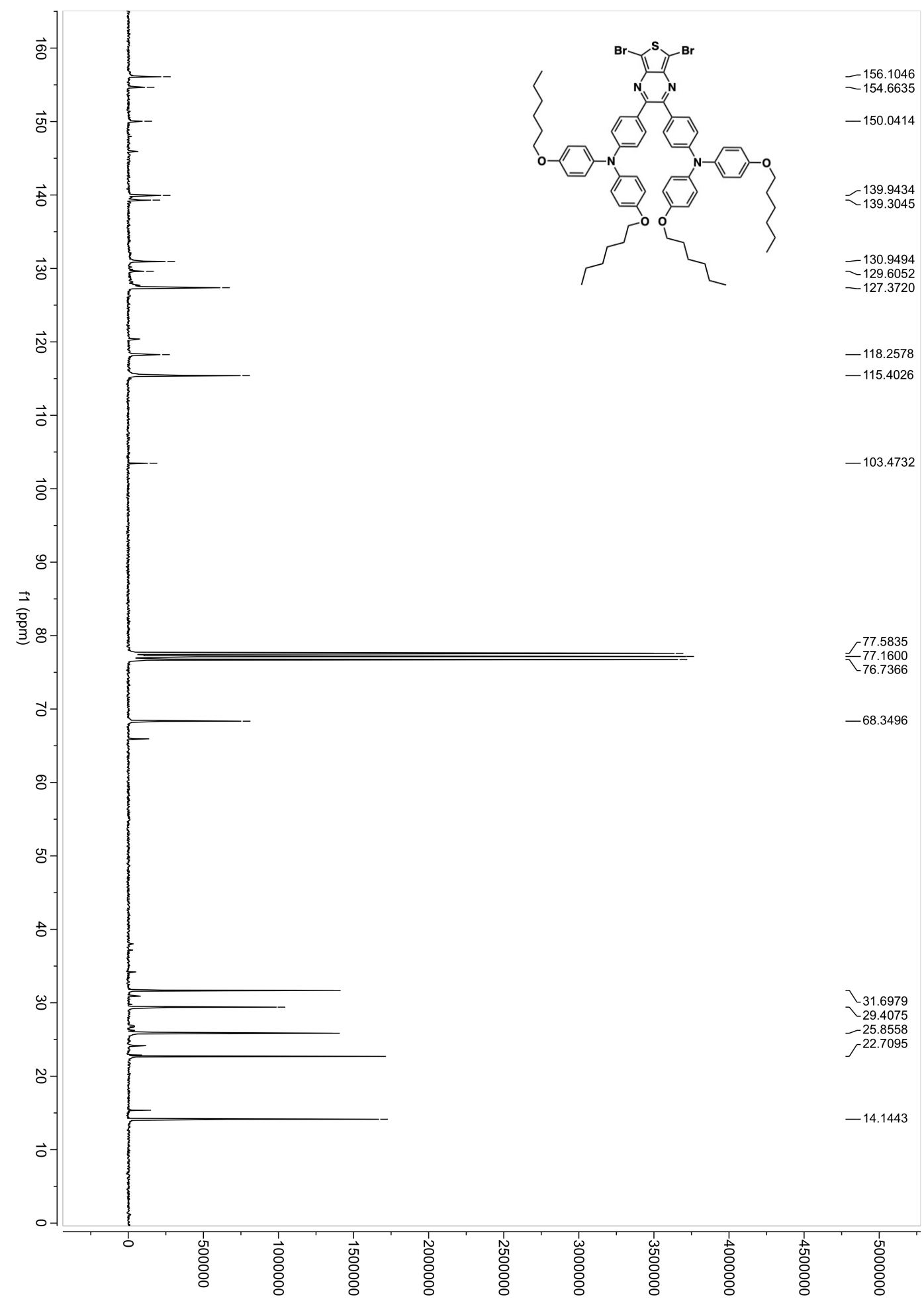

Figure S23. ${ }^{13} \mathrm{C} \mathrm{NMR}\left(\mathrm{CDCl}_{3}\right), 75 \mathrm{MHz}$ (compound 6). 


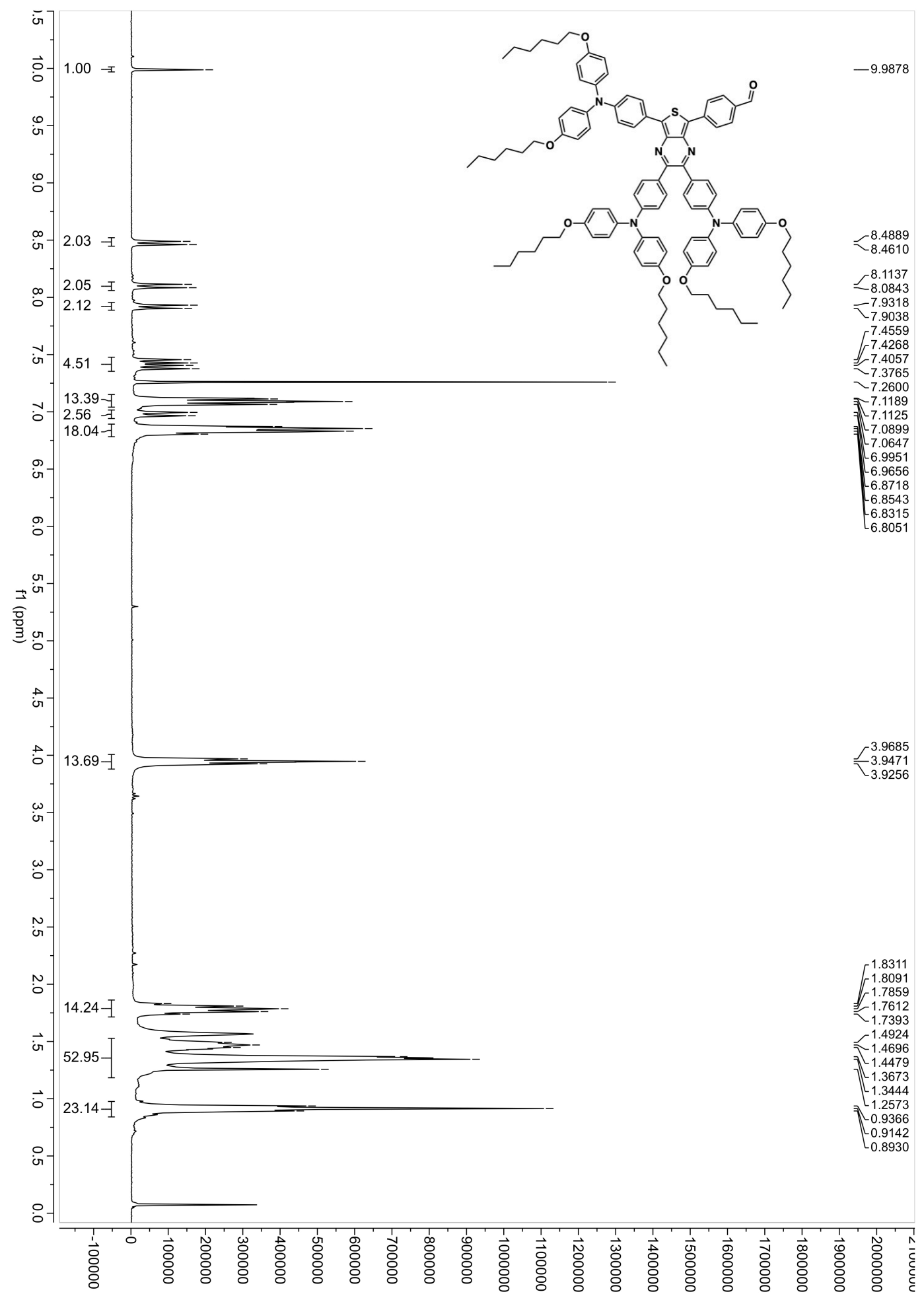

Figure S24. ${ }^{1} \mathrm{H} \mathrm{NMR}\left(\mathrm{CDCl}_{3}\right), 300 \mathrm{MHz}$ (compound 9). 


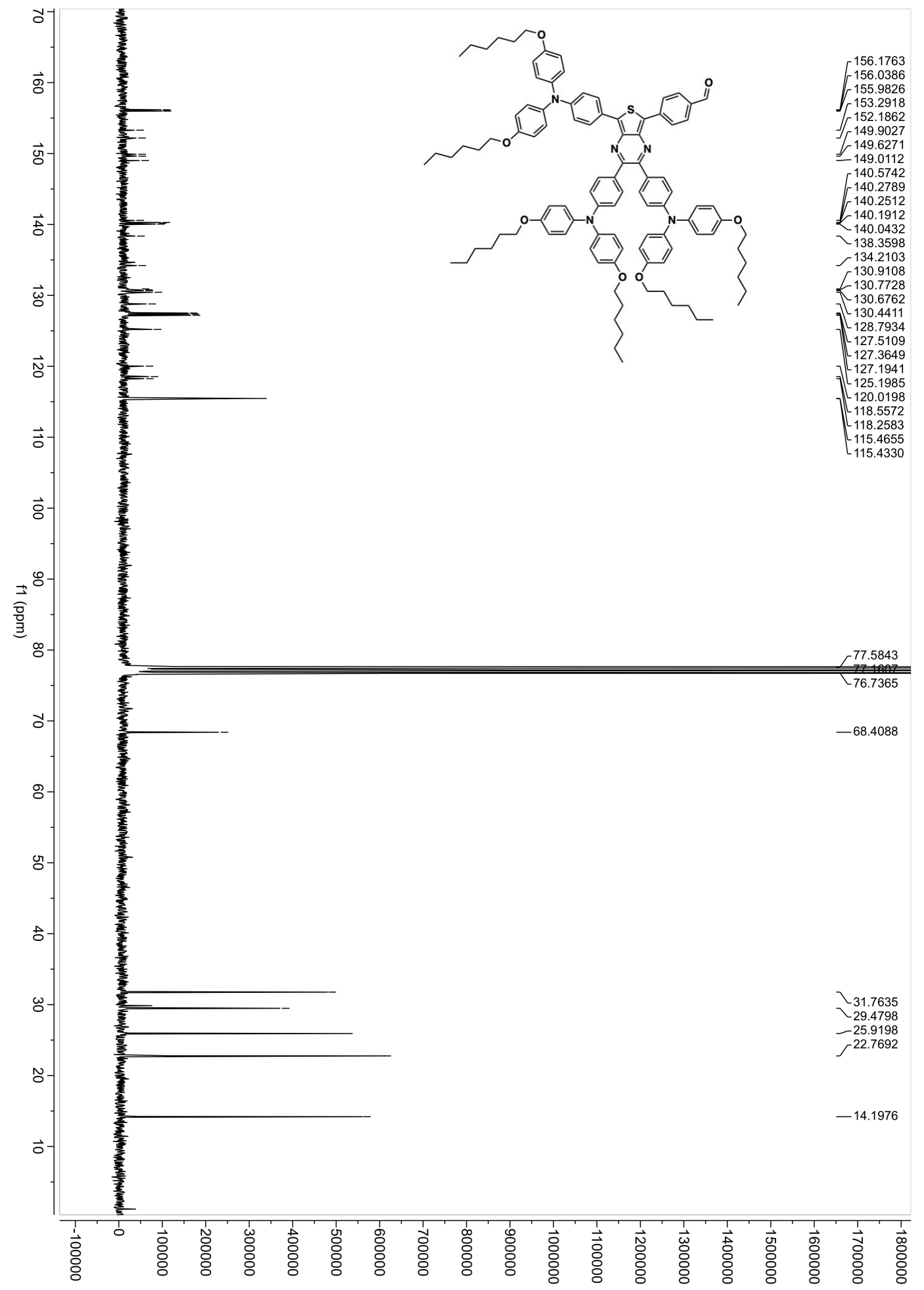

Figure S25. ${ }^{13} \mathrm{C} \mathrm{NMR}\left(\mathrm{CDCl}_{3}\right), 75 \mathrm{MHz}$ (compound 9). 


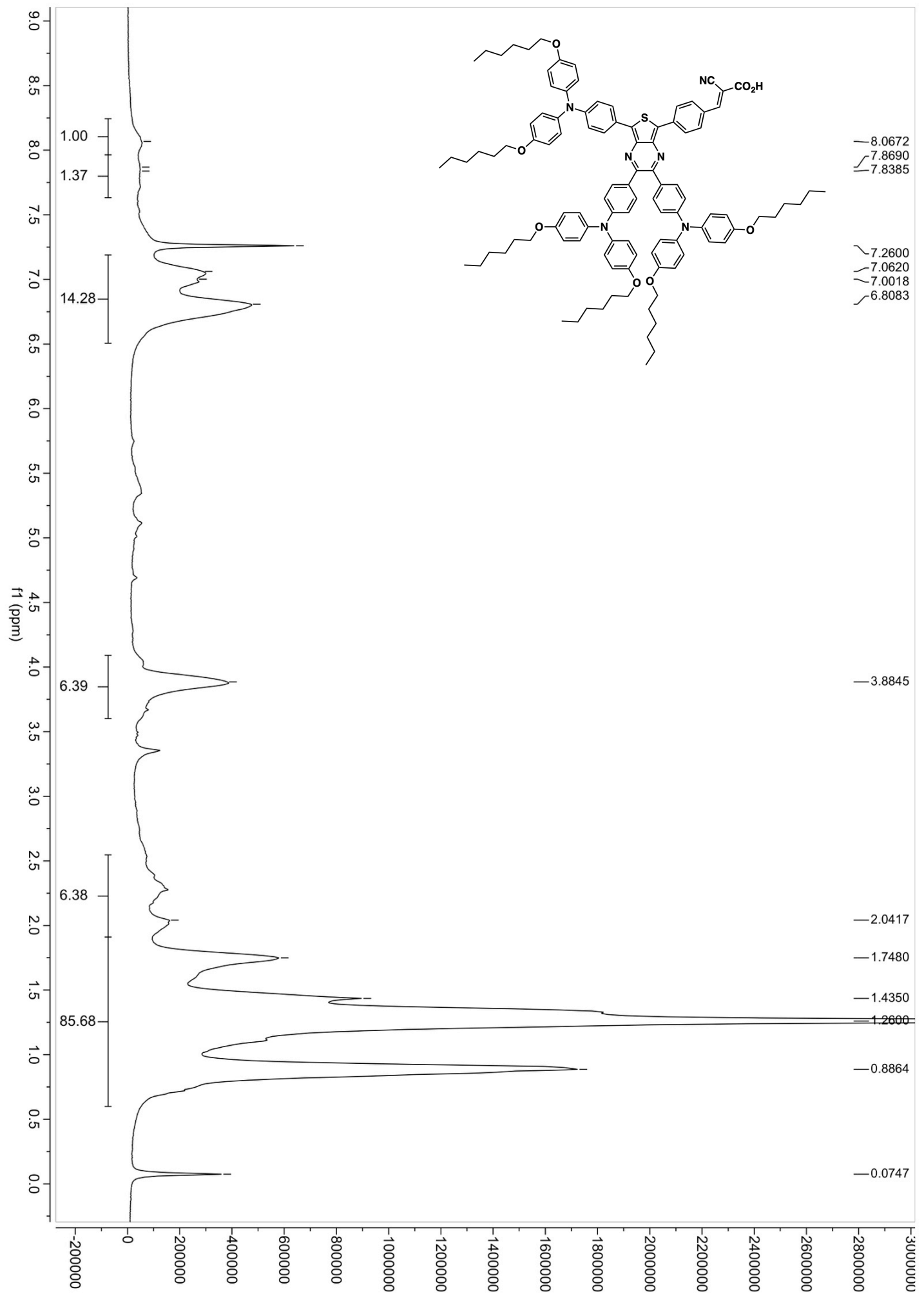

Figure S26. ${ }^{1} \mathrm{H}$ NMR $\left(\mathrm{CDCl}_{3}\right), 300 \mathrm{MHz}(\mathbf{J W} 1)$. The spectrum of JW1 shows considerable dye aggregation once the dye is completely dried. However, if the NMR is taken with acetic acid present (see Figure S27), reasonably resolved peaks are observed. 


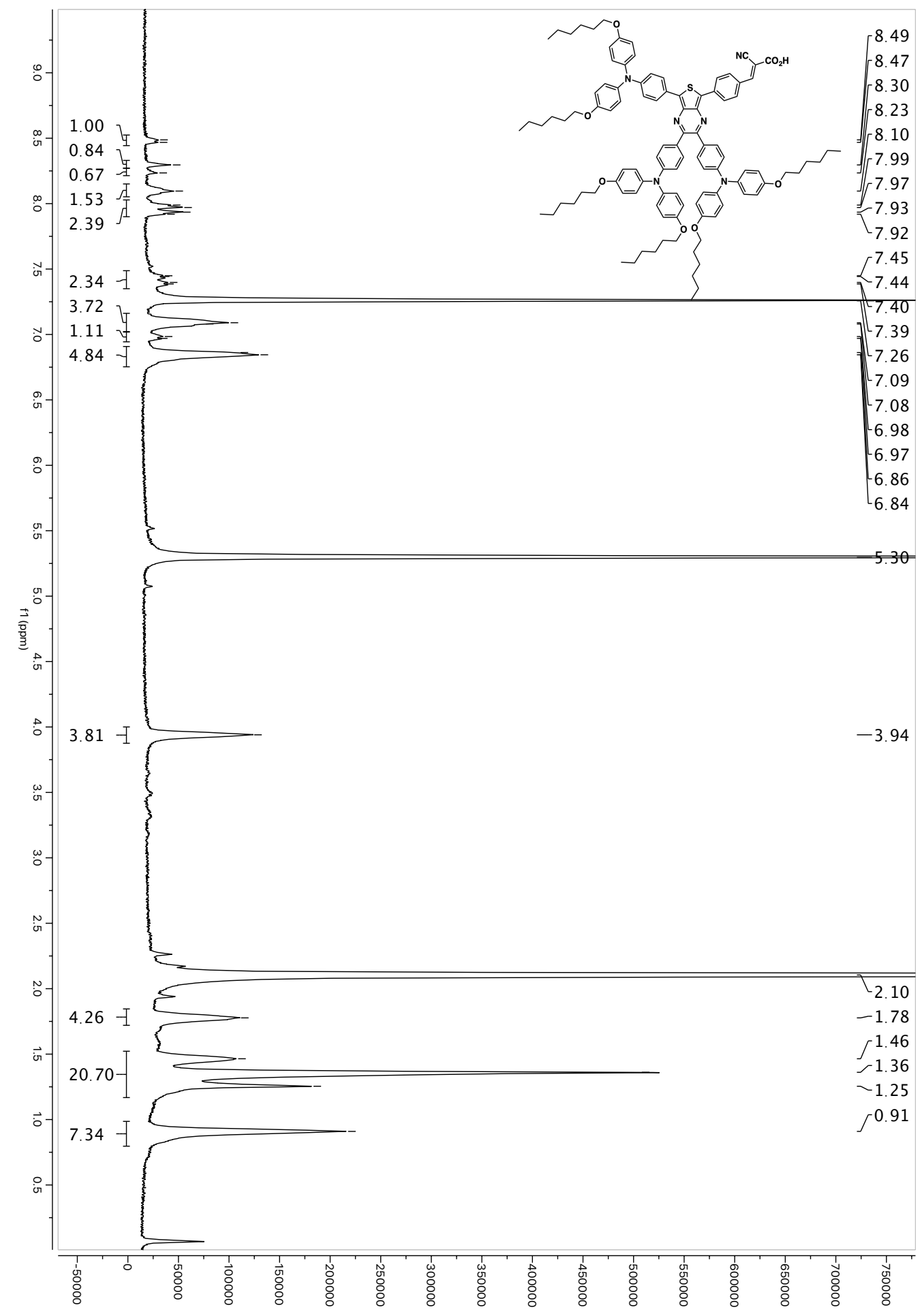

Figure S27. ${ }^{1} \mathrm{H} \mathrm{NMR}\left(\mathrm{CDCl}_{3}+1\right.$ drop of acetic acid), $300 \mathrm{MHz}$ (JW1). Addition of acid was necessary for peak resolution. Variable temperature and changing solvents (acetone or DMSO) did not give well resolved peaks. 